\title{
STABILITY OF TRAVELLING WAVES IN A MODEL FOR CONICAL FLAMES IN TWO SPACE DIMENSIONS
}

\author{
By FRANÇOIS HAMEL, RÉGIS MONNEAU AND \\ JEAN-MICHEL ROQUEJOFFRE
}

\begin{abstract}
This paper deals with the question of the stability of conical-shaped solutions of a class of reaction-diffusion equations in $\mathbb{R}^{2}$. One first proves the existence of travelling waves solutions with conicalshaped level sets, generalizing earlier results by Bonnet, Hamel and Monneau [SIAM J. Math. Anal. 31 (1999) 80-118; Comm. Partial Differential Equations 25 (2000) 769-819]. One then gives a characterization of the global attractor of these semilinear parabolic equations under some conical asymptotic conditions. Lastly, the global stability of the travelling waves solutions is proved.
\end{abstract}

(c) 2004 Elsevier SAS

RÉSUMÉ. - On étudie dans cet article la stabilité des solutions d'ondes progressives à lignes de niveaux coniques pour une classe d'équations de réaction-diffusion dans $\mathbb{R}^{2}$. Nous prouvons d'abord l'existence de telles ondes, généralisant ainsi des résultats antérieurs de Bonnet, Hamel, Monneau [SIAM J. Math. Anal. 31 (1999) 80-118; Comm. Partial Differential Equations 25 (2000) 769-819]. Nous donnons ensuite une caractérisation de l'attracteur global des ces équations paraboliques semi-linéaires sous des contraintes de conditions aux limites coniques équivalentes à celles des ondes progressives. Nous prouvons enfin la stabilité globale des ondes progressives.

(C) 2004 Elsevier SAS

\section{Introduction and main results}

This paper deals with the question of the global stability of the solutions $\phi$ of the following semilinear elliptic equation

$$
\Delta \phi-c \partial_{y} \phi+f(\phi)=0, \quad 0<\phi<1 \quad \text { in } \mathbb{R}^{2},
$$

under the following type of conical conditions at infinity

$$
\left\{\begin{array}{l}
\lim _{y \rightarrow+\infty} \inf _{\mathcal{C}_{+}(y, \pi-\alpha)} \phi=1, \\
\lim _{y \rightarrow-\infty} \sup _{\mathcal{C}_{-}(y, \alpha)} \phi=0 .
\end{array}\right.
$$

Throughout the paper, the notation $\partial_{y} \phi$ (as well as $\phi_{y}$ ) means the partial derivative of the function $\phi$ with respect to the variable $y$. For any $y_{0} \in \mathbb{R}$ and any $0 \leqslant \beta \leqslant \pi$, the lower and upper cones $\mathcal{C}_{ \pm}\left(y_{0}, \beta\right)$ are defined by

$$
\mathcal{C}_{ \pm}\left(y_{0}, \beta\right)=\left\{(x, y)=\left(0, y_{0}\right)+\rho(\cos \varphi, \sin \varphi), \rho \geqslant 0,|\varphi \mp \pi / 2| \leqslant \beta\right\} .
$$




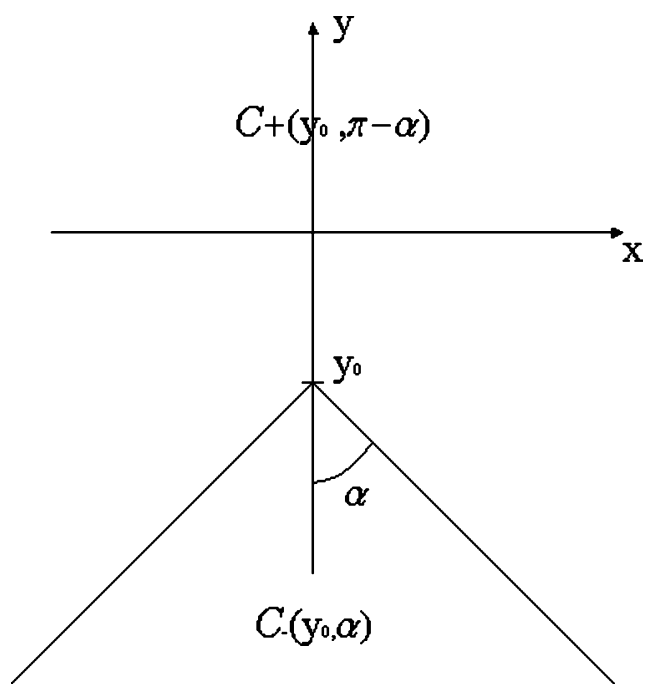

Fig. 1. Upper and lower cones.

We also use the following notation: for a function $v$ of the 2D real variable $(x, y)$, and for $(a, b) \in \mathbb{R}^{2}$, we denote by $\tau_{a, b} v$ the function

$$
\tau_{a, b} v:(x, y) \mapsto v(x+a, y+b) .
$$

Another way of formulating the question of the stability of the solutions $\phi$ of (1.1)-(1.2) is to ask the question of the convergence to the travelling fronts $\phi(x, y+c t)$, or to some translates of them, for the solutions $u(t, x, y)$ of the Cauchy problem

$$
\left\{\begin{array}{l}
u_{t}=\Delta u+f(u), \quad t>0,(x, y) \in \mathbb{R}^{2} \\
u(0, x, y)=u_{0}(x, y) \text { given, } \quad 0 \leqslant u_{0} \leqslant 1
\end{array}\right.
$$

where $u_{0}$ is close, in some sense to be defined later, to a translate $\tau_{a, b} \phi$ of a solution $\phi$ of (1.1)(1.2).

The function $f$ is assumed to be of class $C^{1, \delta}$ in $[0,1]$ (for some $\delta>0$ ) and to have the following profile:

$$
\exists \theta \in(0,1), \quad f=0 \text { on }[0, \theta] \cup\{1\}, \quad f>0 \text { on }(\theta, 1) \quad \text { and } \quad f^{\prime}\left(1^{-}\right)<0 .
$$

For mathematical convenience, we extend $f$ by 0 outside $[0,1]$. Notice that, from standard elliptic estimates, any classical solution $\phi$ of (1.1) is actually of class $C^{2, \mu}\left(\mathbb{R}^{2}\right)$ for any $\mu \in[0,1)$.

Eq. (1.1) arises in models of equidiffusional premixed Bunsen flames. The function $u$ is a normalized temperature and its level sets represent the profile of a conical-shaped Bunsen flame coming out of a thin elongated Bunsen burner (see Buckmaster and Ludford [12], Joulin [24], Sivashinsky [38], Williams [40]). The temperature of the unburnt gases is close to 0 and that of the burnt gases is close to 1 , the hot zone being above the fresh zone. The real $\theta$ is called an ignition temperature, below which no reaction happens. The real $c$ is the speed of the gases at the exit of the burner. Since the shape of the Bunsen flames is invariant with respect to the size of the Bunsen burner, one way of modelling these conical flames consists in setting Eq. (1.1) in the whole plane $\mathbb{R}^{2}$ together with asymptotic conical conditions of the type (1.2). The angle $2 \alpha$ then stands for the aperture of the tip of the flame. 
In the one-dimensional case, Eq. (1.1) and conditions at infinity (1.2) reduce to the ordinary differential equation

$$
\left\{\begin{array}{l}
\phi_{0}^{\prime \prime}-c_{0} \phi_{0}^{\prime}+f\left(\phi_{0}\right)=0, \\
\phi_{0}(-\infty)=0, \quad \phi_{0}(+\infty)=1 .
\end{array}\right.
$$

It is well known (Aronson, Weinberger [2], Berestycki, Nicolaenko, Scheurer [6], Kanel' [25]) that there exists a unique solution $\left(c_{0}, \phi_{0}\right)$ of (1.5) such that $\phi_{0}(0)=\theta$ (the solutions of (1.5) are actually unique up to translation). Besides, the speed $c_{0}$ is positive and the function $\phi_{0}$ is increasing. The function $\phi_{0}(y)$ is also a solution of the two-dimensional problem (1.1)-(1.2) in the particular case $\alpha=\pi / 2$.

In the two-dimensional case with $\alpha \neq \pi / 2$, the existence of solutions $\phi$ of (1.1)-(1.2) was proved by Hamel and Monneau [19] for some angles $\alpha \in(0, \pi / 2)$ and some functions $f$ satisfying (1.4) under some additional assumptions (see Theorem 1.8 in [19]). Existence of solutions of (1.1) under some conical conditions weaker than (1.2) was also proved by Bonnet and Hamel (see Theorem 1.1 in [9]).

The first result of this paper is to establish the existence of solutions of (1.1)-(1.2) for any angle $\alpha \in(0, \pi / 2]$ and for any function $f$ satisfying (1.4):

THEOREM 1.1 (Existence).- For every angle $\alpha \in(0, \pi / 2]$ and for every function $f$ satisfying (1.4), there exists a solution $\phi$ to (1.1)-(1.2), with $c=c_{0} / \sin \alpha$.

Furthermore, it follows from Theorem 1.7 in [19] that the solutions $(c, \phi)$ of (1.1)-(1.2) are unique, in the sense that $c$ is unique, and $\phi$ up to a translation in $(x, y)$. The speed $c$ is necessarily equal to $c=c_{0} / \sin \alpha$. Besides, any solution $\phi$ satisfies the following properties: (1) there exists a real $x_{0}$ such that $\phi$ is symmetric with respect to the vertical line $\left\{x=x_{0}\right\}$, (2) for any $\lambda \in(0,1)$, the level set $\{\phi(x, y)=\lambda\}$ has two asymptots parallel to the half-lines $\{y=-\cot \alpha|x|, x \geqslant 0\}$ and $\{y=-\cot \alpha|x|, x \leqslant 0\}$, (3) there exist two reals $t_{ \pm}$such that for any sequence $x_{n} \rightarrow \pm \infty$, the functions $\phi_{n}(x, y)=\phi\left(x+x_{n}, y-\left|x_{n}\right| \cot \alpha\right)$ go to the planar fronts $\phi_{0}\left( \pm x \cos \alpha+y \sin \alpha+t_{ \pm}\right)$as $x_{n} \rightarrow \pm \infty$ in $C_{\mathrm{loc}}^{2}\left(\mathbb{R}^{2}\right)$. The last two properties mean that any solution $\phi$ is asymptotically conical-shaped far away from the origin: namely, $\phi$ is asymptotically planar and asymptotically equal to two translates of the planar front $\phi_{0}$ in the two directions of angle $\alpha$ with respect to the vector $-e_{2}=(0,-1)$.

The formula $c=c_{0} / \sin \alpha$, which actually follows from earlier results of Bonnet and Hamel [9], and had already been used in several papers (see e.g. Lewis, Von Elbe [28], Sivashinsky [38], Williams [40]), is very natural. Indeed, any solution $\phi$ of (1.1)-(1.2) gives rise to a solution $u(t, x, y)=\phi(x, y+c t)$ of the evolution problem (1.3) with $u_{0}=\phi$. The planar speed $c_{0}$ is now nothing else than the projection on the directions $( \pm \cos \alpha,-\sin \alpha)$ of the vertical speed $c$ of the curved front $\phi(x, y+c t)$ moving downwards. The speed $c_{0}$ is the speed of two planar waves moving in the directions $( \pm \cos \alpha,-\sin \alpha)$ perpendicular to the half-lines making an angle $\alpha$ with the vertical axis.

Remark 1.2. -1 . The dimension 2 is quite different from other dimensions since, as soon as $N \geqslant 3$, there is no solution of problem (1.1) in $\mathbb{R}^{N}$, with $\alpha<\pi / 2$ and conical conditions of the type (1.2) (see [19]). But the possible existence of solutions of (1.1) in $\mathbb{R}^{N}$ under some weaker conical conditions is still open in dimensions $N \geqslant 3$.

2. It was also proved in [19] that no solution of (1.1)-(1.2) exists whenever $\alpha \in(\pi / 2, \pi)$, in dimensions 2 and higher.

Whereas there are many papers dealing with the stability of the travelling fronts for onedimensional equations of the type (1.5) with various types of nonlinearities $f$ (see e.g. [2,10,17, $25,36,37]$ ), or for wrinkled travelling fronts of multidimensional equations in infinite cylinders 
(see [4] and [8] for the existence and uniqueness results, and [5,29,33-35] for the stability results), or lastly for planar fronts in the whole space (see [27,41]), nothing seems to be known about the stability of the solutions of two-dimensional problem (1.1) under conical conditions of the type (1.2), for $\alpha<\pi / 2$. As already emphasized, the travelling fronts $\phi(x, y+c t)$ are special time-global solutions of (1.3) satisfying, at each time, the conical conditions (1.2) in the frame moving downwards with speed $c=c_{0} / \sin \alpha$. Therefore, the question of the global stability of these travelling waves and the question of the asymptotic behaviour for large time of the solutions of the Cauchy problem (1.3) starts from the study of the global attractor of Eq. (1.3) under conical conditions of the type (1.2) in a frame moving downwards with speed $c$.

The next theorem states that the travelling waves are the only time-global solutions of (1.3) satisfying such conical conditions.

THEOREM 1.3 (Liouville type result). - Let $\alpha \in(0, \pi / 2]$ and $0 \leqslant u(t, x, y) \leqslant 1$ solve the equation

$$
u_{t}=\Delta u+f(u), \quad(x, y) \in \mathbb{R}^{2}
$$

with $t \in(-\infty,+\infty)$ and $f$ satisfying (1.4), and assume that

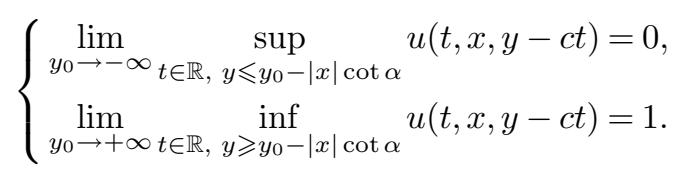

Then there exists a couple $(h, k) \in \mathbb{R}^{2}$ such that $u(t, x, y)=\tau_{h, k} \phi(x, y+c t)$ for all $(t, x, y) \in$ $\mathbb{R} \times \mathbb{R}^{2}$, where $\phi$ is given by Theorem 1.1 .

Since $\phi(x, y) \rightarrow 0$ (respectively $\rightarrow 1$ ) uniformly as $y+|x| \cot \alpha \rightarrow-\infty$ (respectively $y+|x| \cot \alpha \rightarrow+\infty)$, the following corollary holds:

COROLLARY 1.4. - Let $0 \leqslant u(t, x, y) \leqslant 1$ be a solution of (1.6); assume the existence of two couples $\left(a_{1}, b_{1}\right)$ and $\left(a_{2}, b_{2}\right) \in \mathbb{R}^{2}$ for which $\tau_{a_{1}, b_{1}} \phi(x, y+c t) \leqslant u(t, x, y) \leqslant \tau_{a_{2}, b_{2}} \phi(x, y+c t)$ for all $(t, x, y) \in \mathbb{R}^{3}$. Then the conclusion of Theorem 1.3 holds.

The idea for proving Theorem 1.3 is based on a sliding method (see [7]) in the variable $t$ and some versions of the maximum principle for parabolic equations in unbounded domains. Similar methods were used in [35] and [3] to get some monotonicity results for the solutions of some semilinear parabolic equations in various domains.

Theorem 1.3 especially implies the following

THEOREM 1.5 (Convergence of a subsequence to a travelling wave). - Let $\phi$ be a solution of (1.1)-(1.2) for $\alpha \in(0, \pi / 2]$ with assumptions (1.4) on $f$. Let $u(t, x, y)$ be a solution of the Cauchy problem (1.3) such that

$$
\left\{\begin{array}{l}
u_{0} \leqslant \phi \quad \text { in } \mathbb{R}^{2} \\
\lim _{y_{0} \rightarrow+\infty} \inf _{y \geqslant y_{0}-|x| \cot \alpha} u_{0}(x, y)>\theta .
\end{array}\right.
$$

Then, for every sequence $t_{n} \rightarrow+\infty$, there exist a subsequence $t_{n^{\prime}} \rightarrow+\infty$ and $(a, b) \in \mathbb{R}^{2}$ such that

$$
u\left(t_{n^{\prime}}+t, x, y-c t_{n^{\prime}}-c t\right) \rightarrow \phi(x+a, y+b) \quad \text { as } n^{\prime} \rightarrow+\infty
$$

locally uniformly in $(t, x, y) \in \mathbb{R}^{3}$. 
A consequence of this result is that, if $u_{0}$ satisfies (1.8) and if $\omega\left(u_{0}\right)$ is the $\omega$-limit set of $u_{0}$ for the semi-group $\mathcal{S}(t)$ given by (1.3), then $\omega\left(u_{0}\right)$ is made up of travelling waves. Condition (1.8) is especially satisfied when $u_{0}$ lies between two translates of a solution $\phi$ of (1.1)-(1.2). But, even under condition (1.8), the $\omega$-limit set $\omega\left(u_{0}\right)$ of $u_{0}$ may well be a continuum, and one may ask for sufficient conditions for $\omega\left(u_{0}\right)$ to be a singleton. This is the goal of Theorem 1.6 below.

Before stating this result, let us first introduce some notations. Denote by $U C\left(\mathbb{R}^{2}\right)$ the space of all bounded uniformly continuous functions from $\mathbb{R}^{2}$ to $\mathbb{R}$. We fix a $C^{\infty}$ function $g: \mathbb{R} \rightarrow \mathbb{R}$ such that $g(x)=|x|$ for $|x|$ large enough. For $\rho>0$, we set

$$
q(x, y)=e^{-\rho(g(x) \sin \alpha-y \cos \alpha)}
$$

and

$$
G_{\rho}=\left\{w \in U C\left(\mathbb{R}^{2}\right), \limsup _{|(x, y)| \rightarrow+\infty}|w(x, y)|=0, w / q \in L^{\infty}\left(\mathbb{R}^{2}\right)\right\} .
$$

The space $G_{\rho}$ is a Banach space with the norm

$$
\|w\|_{G_{\rho}}=\|w\|_{L^{\infty}\left(\mathbb{R}^{2}\right)}+\|w / q\|_{L^{\infty}\left(\mathbb{R}^{2}\right)} .
$$

THEOREM 1.6 (Stability result). - Choose $\alpha \in(0, \pi / 2)$ and let $f$ satisfy (1.4). Let $u(t, x, y)$ be a solution of the Cauchy problem (1.3) with initial datum $u_{0} \in U C\left(\mathbb{R}^{2}\right)$ such that $0 \leqslant u_{0} \leqslant 1$. Assume the existence of $\rho_{0}, C_{0}>0$ and of a solution $\phi$ of (1.1)-(1.2) such that

$$
\left|u_{0}(x, y)-\phi(x, y)\right| \leqslant C_{0} e^{-\rho_{0} \sqrt{x^{2}+y^{2}}}
$$

in $\mathbb{R}^{2}$. Also assume that there exists $(a, b) \in \mathbb{R}^{2}$ such that $u_{0} \leqslant \tau_{a, b} \phi$ in $\mathbb{R}^{2}$.

Then there are four constants $T \geqslant 0, K \geqslant 0, \omega>0$ and $\rho>0$, such that

$$
\forall t \geqslant T, \quad\|u(t, x, y-c t)-\phi(x, y)\|_{G_{\rho}} \leqslant K e^{-\omega t} .
$$

Under the above assumptions, it especially follows that $u(t, \cdot, \cdot-c t)$ converges to $\phi$ uniformly in $\mathbb{R}^{2}$, and exponentially in time. Notice also that if $\left|u_{0}-\phi\right| \leqslant C_{0} e^{-\rho_{0} \sqrt{x^{2}+y^{2}}}$ in $\mathbb{R}^{2}$ for some solution $\phi$ of (1.1)-(1.2), then $u_{0}$ and $\phi$ have the same limits along the lines $y=-|x| \cot \alpha$ as $x \rightarrow \pm \infty$, whence such a $\phi$, if any, is unique.

Notice that Theorem 1.6 holds especially if $u_{0} \in U C\left(\mathbb{R}^{2}\right)$ is such that, say, $0 \leqslant u_{0}<1$ and if there exists a solution $\phi$ of (1.1)-(1.2) such that $u_{0}-\phi$ has compact support.

Lastly, the following theorem holds:

THEOREM 1.7. - Let $\alpha \in(0, \pi / 2)$, and $f$ satisfy (1.4). Let $0 \leqslant u(t, x, y) \leqslant 1$ be a solution of the Cauchy problem (1.3) with $u_{0}$ bounded in $C^{1}\left(\mathbb{R}^{2}\right)$ and $0 \leqslant u_{0} \leqslant 1$. Assume that $\lim _{y \rightarrow+\infty} \inf _{\mathcal{C}_{+}(y, \pi-\alpha)} u_{0}>\theta$ and that there exists a solution $\phi$ of (1.1)-(1.2) such that $u_{0} \leqslant \phi$ in $\mathbb{R}^{2}$. Also assume that for some $\rho_{0}>0$

$$
\left|\partial_{e_{\alpha}} u_{0}(x, y)\right| \leqslant C e^{\rho_{0}(y \sin \alpha-x \cos \alpha)}, \quad\left|\partial_{e_{\alpha}^{\prime}} u_{0}(x, y)\right| \leqslant C e^{\rho_{0}(y \sin \alpha+x \cos \alpha)}
$$

for all $(x, y) \in \mathbb{R}^{2}$, where

$$
e_{\alpha}=(\sin \alpha,-\cos \alpha) \quad \text { and } \quad e_{\alpha}^{\prime}=(-\sin \alpha,-\cos \alpha) .
$$

Then the function $u(t, \cdot, \cdot-c t)$ converges uniformly in $\mathbb{R}^{2}$, as $t \rightarrow+\infty$, to a solution $\phi^{\prime}$ of (1.1)-(1.2). 
Remark 1.8. - The convergence phenomenon is really governed by the behaviour of the initial datum when the space variable becomes infinite along the directions $e_{\alpha}$ and $e_{\alpha}^{\prime}$. In that sense, the situation is similar to the KPP situation; see [29]. It may well happen that, if the initial datum $u_{0}$ has no limit in the $e_{\alpha}$ and $e_{\alpha}^{\prime}$ directions, its $\omega$-limit is made up of a continuum of waves (see [15]).

Let us mention here similar stability results were obtained by Ninomiya and Taniguchi [32] for curved fronts in singular limits for Allen-Cahn bistable equations. Existence of smooth solutions of problem (1.1)-(1.2) with bistable nonlinearity $f$ was obtained by Fife [16] for angles $\alpha<\pi / 2$ close to $\pi / 2$. Conical-shaped and more general curved fronts also exist for the Fisher-KPP equation, with concave nonlinearity $f$ (see $[11,21]$ ). Other stability results were also obtained by Michelson [31] for Bunsen fronts solving the Kuramoto-Sivashinsky equation, in some asymptotic regimes. Formal stability results in the nearly equidiffusional case were also given in [30].

The plan of the paper is the following. Section 2 is devoted to the proof of the existence of travelling waves with the conical conditions at infinity. In Section 3, we prove that global solutions - i.e. defined for all $t \in \mathbb{R}$ - are travelling wave solutions. In order to prove Theorem 1.6, we present a local stability result in Section 4; combined to Section 3, this implies the global stability: this last item will be treated in Section 5.

\section{Existence of travelling wave solutions}

\subsection{Proof of Theorem 1.1}

Let $\alpha \in(0, \pi / 2]$ be given. We are looking for a solution $\phi$ of (1.1), i.e.

$$
\Delta \phi-c \partial_{y} \phi+f(\phi)=0, \quad 0<\phi<1 \quad \text { in } \mathbb{R}^{2}
$$

with $c=c_{0} / \sin \alpha$, satisfying the conditions (1.2) at infinity, i.e.

$$
\left\{\begin{array}{l}
\lim _{y_{0} \rightarrow+\infty} \inf _{y \geqslant y_{0}-|x| \cot \alpha} \phi(x, y)=1 \\
\lim _{y_{0} \rightarrow-\infty} \sup _{y \leqslant y_{0}-|x| \cot \alpha} \phi(x, y)=0
\end{array}\right.
$$

The strategy to prove Theorem 1.1 is to build a solution $\phi$ between a sub- and a supersolution in the whole plane $\mathbb{R}^{2}$.

We perform the proof in three steps.

Step 1: Construction of a subsolution. A natural candidate for a subsolution is the following function:

$$
\underline{\phi}(x, y)=\phi_{0}\left(\left(y-\gamma_{0}(x)\right) \sin \alpha\right)
$$

where

$$
\gamma_{0}(x)=-\frac{1}{c_{0} \sin \alpha} \ln \left(\cosh \left(x c_{0} \cos \alpha\right)\right),
$$

and $\phi_{0}$ is the solution of the one-dimensional problem (1.5) satisfying $\phi_{0}(0)=\theta$. It can easily be checked (see also [19] where such subsolutions were used) that $\underline{\phi}$ is a classical subsolution of

$$
\Delta \underline{\phi}-c \partial_{y} \underline{\phi}+f(\underline{\phi})=\frac{\cos ^{2} \alpha}{\cosh ^{2}\left(x c_{0} \cos \alpha\right)} f\left(\phi_{0}\left(\left(y-\gamma_{0}(x)\right) \sin \alpha\right)\right) \geqslant 0 \quad \text { in } \mathbb{R}^{2} .
$$

Furthermore, $\underline{\phi}$ is a solution of $\Delta \underline{\phi}-c \partial_{y} \underline{\phi}=0$ in $\left\{y \leqslant \gamma_{0}(x)\right\}$. Notice that since $\underline{\phi}$ is of class $C^{2}$, it is also a subsolution of $\Delta \underline{\phi}-\bar{c}_{y} \underline{\phi}+\bar{f}(\underline{\phi}) \geqslant 0$ in the viscosity sense. 
Moreover, the function $\gamma_{0}$ satisfies $\sup _{x \in \mathbb{R}}\left|\gamma_{0}(x)+\right| x|\cot \alpha|<+\infty$. This implies in particular

$$
\lim _{y_{0} \rightarrow-\infty} \sup _{\left\{y \leqslant y_{0}-|x| \cot \alpha\right\}} \underline{\phi}(x, y)=0
$$

and

$$
\lim _{y_{0} \rightarrow+\infty} \inf _{\left\{y \geqslant y_{0}-|x| \cot \alpha\right\}} \phi(x, y)=1 .
$$

Step 2: Construction of a supersolution. On the contrary, the construction of a supersolution which is above the subsolution is a nontrivial fact, and requires the use of the solution $\psi$ to an associated free boundary problem.

We define the candidate for the supersolution as:

$$
\bar{\phi}(x, y)=\left\{\begin{array}{l}
\theta \psi(x, y) \quad \text { in } \Omega:=\{\psi<1\}, \\
\phi_{0}(\operatorname{dist}((x, y), \Omega)) \quad \text { in } \mathbb{R}^{2} \backslash \Omega
\end{array}\right.
$$

where dist denotes the euclidean distance function and $\psi$ is the unique (up to shift) solution to the following free boundary problem (see [20]):

THEOREM 2.1 (A free boundary problem, ${ }^{1}$ [20]). - For $\alpha \in(0, \pi / 2], c_{0}>0$ and $c=c_{0} / \sin \alpha$, there exists a function $\psi$ satisfying

$$
\left\{\begin{array}{l}
\Delta \psi-c \partial_{y} \psi=0 \quad \text { in } \Omega:=\{\psi<1\} \\
0<\psi \leqslant 1 \quad \text { in } \mathbb{R}^{2} \\
\frac{\partial \psi}{\partial n}=c_{0} \quad \text { on } \Gamma:=\partial \Omega \\
\lim _{y \rightarrow-\infty} \sup _{\mathcal{C}^{-}(y, \alpha)} \psi=0 \\
\psi=1 \quad \text { in } \mathcal{C}^{+}\left(y_{0}, \pi-\alpha\right) \text { for some } y_{0} \in \mathbb{R}
\end{array}\right.
$$

where $\frac{\partial \psi}{\partial n}$ stands for the normal derivative on $\Gamma$ of the restriction of $\psi$ to $\bar{\Omega}$. Furthermore, $\psi$ is continuous in $\mathbb{R}^{2}$, the set $\Gamma=\partial \Omega$ is a $C^{\infty}$ graph $\Gamma=\{y=\varphi(x), x \in \mathbb{R}\}$ such that

$$
\sup _{x \in \mathbb{R}}|\varphi(x)+| x|\cot \alpha|<+\infty
$$

$\Omega$ is the subgraph $\Omega=\{y<\varphi(x)\}$, the restriction of $\psi$ is $C^{\infty}$ in $\bar{\Omega}$, and $\left|\varphi^{\prime}(x)\right| \leqslant \cot \alpha$ in $\mathbb{R}$. Lastly, $\psi$ is nondecreasing in $y$, even in $x$ and satisfies

$$
\partial_{x} \psi(x, y) \geqslant 0 \text { for } x \geqslant 0, y<\varphi(x) .
$$

From Theorem 2.1 and from the definition of $\gamma_{0}$, it is easy to see that there exist two positive constants $r_{0}$ and $C$ such that

$$
\forall r \geqslant r_{0}, \quad \underline{\phi}^{r}(x, y):=\underline{\phi}(x, y-r) \leqslant \theta \quad \text { in } \bar{\Omega}
$$

and

\footnotetext{
${ }^{1}$ This problem arises in models of equidiffusional premixed Bunsen flames in the limit of high activation energy. The existence of a solution $\psi$ of problem (2.3) can be obtained by regularizing approximations, starting from solutions of problems of the type (1.1) with nonlinearities $f_{\varepsilon}$ approximating a Dirac mass at 1 .
} 


$$
\operatorname{dist}((x, y), \Omega)) \geqslant-C+\left(y-\gamma_{0}(x)\right) \sin \alpha \quad \text { in } \mathbb{R}^{2} \backslash \Omega=\{\psi=1\} .
$$

Because of (2.1), and from the comparison principles proved in [19], it follows that $\underline{\phi}^{r} \leqslant \bar{\phi}$ in $\Omega$ for all $r \geqslant r_{0}$ and then, by construction of $\bar{\phi}$, we get that

$$
\underline{\phi}^{r} \leqslant \bar{\phi} \quad \text { in } \mathbb{R}^{2}
$$

as soon as $r \geqslant \max \left(r_{0}, C / \sin \alpha\right)$.

Moreover, notice that the construction of $\bar{\phi}$ implies that

$$
\lim _{y_{0} \rightarrow-\infty} \sup _{\left\{y \leqslant y_{0}-|x| \cot \alpha\right\}} \bar{\phi}(x, y)=0 .
$$

We shall prove in Section 2.2 the following result.

PROPOSITION 2.2. - The function $\bar{\phi}$ is a supersolution of (1.1) in the vicosity sense.

Step 3. Existence of a solution. Choose a real number $r$ such that $r \geqslant \max \left(r_{0}, C / \sin \alpha\right)$. By using the Perron method for viscosity solutions (see [14] and H. Ishii [23], Theorem 7.2, p. 41), we get the existence of a vicosity solution $\phi$ of $\Delta \phi-c \partial_{y} \phi+f(\phi)=0$, which satisfies:

$$
0 \leqslant \underline{\phi}^{r} \leqslant \phi \leqslant \bar{\phi} \leqslant 1 \quad \text { in } \mathbb{R}^{2} .
$$

Now by the regularity theory for viscosity solutions (see [13]), it follows that $\phi$ is $C^{2+\beta}$ (with $\beta>0$ ), and then $\phi$ is a classical solution of (1.1). Finally $\phi$ satisfies the conditions at infinity (1.2) because of (2.2) and (2.4). This completes the proof of Theorem 1.1.

\subsection{Proof of Proposition 2.2}

The proof of Proposition 2.2 is based on the following result:

LEMMA 2.3. - Let $\xi$ be the function defined by

$$
\xi(x, y)=\phi_{0}^{-1}(\theta \psi(x, y)) \quad \text { in } \bar{\Omega}=\{y \leqslant \varphi(x)\},
$$

where $\psi$ is the solution to the free boundary problem given by Theorem 2.1. Then

$$
|\nabla \xi| \leqslant 1 \quad \text { in } \bar{\Omega} \text {. }
$$

Proof. - We have

$$
\left\{\begin{array}{l}
\Delta \xi+c_{0}\left(|\nabla \xi|^{2}-\frac{\partial_{y} \xi}{\sin \alpha}\right)=0 \quad \text { in } \Omega=\{\xi<0\} \\
\xi=0 \text { and } \frac{\partial \xi}{\partial n}=1 \quad \text { on } \Gamma=\partial\{\xi<0\}
\end{array}\right.
$$

since $\phi_{0}(s)=\theta e^{c_{0} s}$ for all $s \leqslant 0$. A straightforward computation gives, for $v=|\nabla \xi|^{2}$ :

$$
\Delta v+b \cdot \nabla v=2\left|D^{2} \xi\right|^{2},
$$

where $b=2 c_{0} \nabla \xi-c_{0} / \sin \alpha e_{y}$ and $e_{y}=(0,1)$.

Let us define $M=\sup v$. We want to prove that $M \leqslant 1$. Let us assume that $M>1$. We know that $v=1$ on $\Gamma$ and $v(x, y) \rightarrow 1$ as $|x| \rightarrow+\infty$ and $d((x, y), \Gamma)$ stays bounded. From 
the maximum principle we conclude that there exists a sequence of points $\left(x_{n}, y_{n}\right)$ such that $v\left(x_{n}, y_{n}\right) \rightarrow M, d\left(\left(x_{n}, y_{n}\right), \Gamma\right) \rightarrow+\infty$, and the sequence of functions

$$
v_{n}(x, y)=v\left(x_{n}+x, y_{n}+y\right)
$$

converges to the function $v_{\infty}(x, y)$ which from the strong maximum principle satisfies $v_{\infty}(x, y) \equiv M$. Moreover $\xi_{0, n}(x, y)=\xi_{0, n}\left(x_{n}+x, y_{n}+y\right)-\xi_{0, n}\left(x_{n}, y_{n}\right)$ converges to a function $\xi_{0, \infty}(x, y)$ such that $v_{\infty}=\left|\nabla \xi_{0, \infty}\right|^{2}$, and $D^{2} \xi_{0, \infty} \equiv 0$.

On the other hand, the following function

$$
w(x, y)=e^{c_{0}(y \sin \alpha+x \cos \alpha)}+e^{c_{0}(y \sin \alpha-x \cos \alpha)}=e^{\left.c_{0} \sin \alpha(y-g(x))\right)}
$$

is a solution of the equation $\Delta w-\frac{c_{0}}{\sin \alpha} \partial_{y} w=0$ on the whole space. Using the comparison principle on the Lipschitz subgraph $\Omega=\{y<\varphi(x)\}$, we can deduce that there exist two constants $y_{1}>y_{2}$ such that

$$
e^{\left.c_{0} \sin \alpha\left(y-g(x)-y_{1}\right)\right)} \leqslant \psi \leqslant e^{\left.c_{0} \sin \alpha\left(y-g(x)-y_{2}\right)\right)} \quad \text { on } \Omega
$$

and then a simple computation implies

$$
\left|\xi_{0, \infty}(x, y)\right| \leqslant\left|y_{1}-y_{2}\right| \sin \alpha+\sqrt{x^{2}+y^{2}} .
$$

Because $M>1$, this is in contradiction with $\nabla \xi_{0, \infty} \equiv \nu \sqrt{M}$ for a constant vector $\nu$ satisfying $\|\nu\|=1$. This ends the proof of the lemma.

Let us now turn to the

Proof of Proposition 2.2. - Let us define

$$
I[u]:=\Delta u-c \partial_{y} u+f(u) .
$$

By construction $\bar{\phi}$ is a classical solution of $I[\bar{\phi}]=0$ in the open set $\Omega=\{\bar{\phi}<\theta\}$. Moreover the gradient of $\bar{\phi}$ is continuous across $\Gamma=\partial\{\bar{\phi}<\theta\}$, which is smooth.

Let us now consider the function $\xi(x, y)=\phi_{0}^{-1}(\bar{\phi}(x, y))$, defined in the whole plane $\mathbb{R}^{2}$. We have

$$
J[\xi]:=\frac{I[\bar{\phi}]}{\phi_{0}^{\prime}(\xi)}=\Delta \xi+c_{0}\left(|\nabla \xi|^{2}-\frac{\partial_{y} \xi}{\sin \alpha}\right)+G(\xi)\left(1-|\nabla \xi|^{2}\right)
$$

in the viscosity sense in $\mathbb{R}^{2}$, where $G(\xi)=f\left(\phi_{0}(\xi)\right) / \phi_{0}^{\prime}(\xi) \geqslant 0$. Because $\xi(x, y)=d((x, y), \Gamma)$ in $\mathbb{R}^{2} \backslash \Omega=\{\xi \geqslant 0\}$, the following inequality holds in the viscosity sense:

$$
J[\xi] \leqslant H[\xi]:=-\frac{K}{1-K \xi}+c_{0}\left(1-\frac{n \cdot e_{y}}{\sin \alpha}\right) \quad \text { in }\{y>\varphi(x)\},
$$

and equality holds where $\xi$ is smooth (see Gilbarg, Trudinger [18]). Here $K=K(Y)$ and $n=n(Y)$ are respectively the curvature ${ }^{2}$ and the exterior normal to the set $\bar{\Omega}$ at a point $Y=Y(x, y) \in \Gamma$ where the ball $B_{\xi(x, y)}((x, y))$ is tangent to $\Gamma$.

On the other hand, on the level set $\Gamma$ we have $|\nabla \xi|=1$ and because of Lemma 2.3 we get $D_{n n}^{2} \xi \geqslant 0$. Therefore, since $I[\bar{\phi}]=0$ in $\bar{\Omega}$, we deduce from (2.5) that

\footnotetext{
${ }^{2}$ Under the convention that the curvature of a disk is negative.
} 


$$
-K(Y)+c_{0}\left(1-\frac{n(Y) \cdot e_{y}}{\sin \alpha}\right) \leqslant 0 \quad \text { for all } Y \in \Gamma .
$$

Furthermore, observe that the inequality

$$
\frac{-K(Y)}{1-K(Y) \xi(x, y)} \leqslant-K(Y)
$$

holds for all $(x, y) \in \mathbb{R}^{2} \backslash \Omega=\{\xi \geqslant 0\}$, whatever the sign of $K$ is, under the same notations as above for $Y$.

Therefore, $H[\xi] \leqslant 0$ in $\mathbb{R}^{2} \backslash \Omega$ and finally $J[\xi] \leqslant 0$ in $\{y>\varphi(x)\}=\{\xi>0\}$ in the viscosity sense. Hence, $I[\bar{\phi}] \leqslant 0$ in $\mathbb{R}^{2}$ in the viscosity sense, which ends the proof of Proposition 2.2.

\section{Global solutions are travelling waves}

This section is devoted to the proof of Theorems 1.3 and 1.5 below, the latter being a consequence of the former.

One of the main tools in the proof of Theorem 1.3 is the following comparison principle:

Proposition 3.1 (Comparison principle).- Let $\delta \in \mathbb{R}$ and $g: \mathbb{R} \rightarrow \mathbb{R}$ be a Lipschitzcontinuous function which is nonincreasing in $(-\infty, \delta]$. Let $\psi: \mathbb{R} \rightarrow \mathbb{R}$ be a Lipschitz-continuous function. Let $\underline{v}:(t, x, y) \mapsto \underline{v}(t, x, y)$ and $\bar{v}:(t, x, y) \mapsto \bar{v}(t, x, y)$ be two bounded and Lipschitzcontinuous functions defined on $\mathbb{R} \times \bar{\Omega}$, where $\Omega=\{y<\psi(x)\}$. Let $\kappa \in \mathbb{R}$. Assume that

$$
\begin{cases}\underline{v}_{t} \leqslant \Delta \underline{v}+\kappa \partial_{y} \underline{v}+g(\underline{v}) & \text { in } \mathcal{D}^{\prime}(\mathbb{R} \times \Omega), \\ \bar{v}_{t} \geqslant \Delta \bar{v}+\kappa \partial_{y} \bar{v}+g(\bar{v}) & \text { in } \mathcal{D}^{\prime}(\mathbb{R} \times \Omega),\end{cases}
$$

$\underline{v} \leqslant \delta$ in $\mathbb{R} \times \bar{\Omega}, \underline{v}(t, x, \psi(x)) \leqslant \bar{v}(t, x, \psi(x))$ for all $(t, x) \in \mathbb{R}^{2}$, and

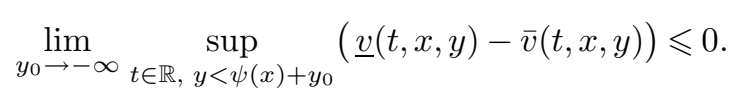

Then $\underline{v}(t, x, y) \leqslant \bar{v}(t, x, y)$ for all $(t, x, y) \in \mathbb{R} \times \bar{\Omega}$.

Proof. - Since $\underline{v}$ and $\bar{v}$ are bounded in $\mathbb{R} \times \bar{\Omega}$, one has $\underline{v}-\varepsilon \leqslant \bar{v}$ in $\mathbb{R} \times \bar{\Omega}$ for $\varepsilon>0$ large enough. Let us now define

$$
\varepsilon^{*}=\inf \left\{\varepsilon>0, \underline{v}-\varepsilon^{\prime} \leqslant \bar{v} \text { in } \mathbb{R} \times \bar{\Omega} \text { for all } \varepsilon^{\prime} \geqslant \varepsilon\right\} .
$$

By continuity, one can immediately say that $\underline{v}-\varepsilon^{*} \leqslant \bar{v}$ in $\mathbb{R} \times \bar{\Omega}$.

Let us now assume that $\varepsilon^{*}>0$. There exist then a sequence $\varepsilon_{n} \leftrightharpoons \varepsilon^{*}$ and a sequence of points $\left(t_{n}, x_{n}, y_{n}\right)$ in $\mathbb{R} \times \bar{\Omega}$ such that

$$
\underline{v}\left(t_{n}, x_{n}, y_{n}\right)-\varepsilon_{n}>\bar{v}\left(t_{n}, x_{n}, y_{n}\right) .
$$

Since $\varepsilon_{n} \geqslant \varepsilon^{*} / 2>0$ for $n$ large enough, it follows from the assumptions of Proposition 3.1 that there exist two real numbers $0<A \leqslant B$ such that

$$
\psi\left(x_{n}\right)-B \leqslant y_{n} \leqslant \psi\left(x_{n}\right)-A
$$

for $n$ large enough. 
Call $\psi_{n}(x)=\psi\left(x+x_{n}\right)-y_{n}$ and let $\underline{v}_{n}$ and $\bar{v}_{n}$ the functions defined in $\mathbb{R} \times\left\{y \leqslant \psi_{n}(x)\right\}$ by

$$
\underline{v}_{n}(t, x, y)=\underline{v}\left(t+t_{n}, x+x_{n}, y+y_{n}\right) \quad \text { and } \quad \bar{v}_{n}(t, x, y)=\bar{v}\left(t+t_{n}, x+x_{n}, y+y_{n}\right) .
$$

Since the functions $\psi_{n}$ are uniformly Lipschitz-continuous, they locally converge, up to extraction of some subsequence, to a globally Lipschitz-continuous function $\psi_{\infty}$. Similarly, up to extraction of another subsequence, the functions $\underline{v}_{n}$ and $\bar{v}_{n}$ converge locally uniformly in $\mathbb{R} \times\left\{y<\psi_{\infty}(x)\right\}$ to two globally Lipschitz-continuous functions $\underline{v}_{\infty}$ and $\bar{v}_{\infty}$, which can be extended by continuity on $\mathbb{R} \times\left\{y=\psi_{\infty}(x)\right\}$. Call

$$
\Omega_{\infty}=\left\{(x, y) \in \mathbb{R}^{2}, y<\psi_{\infty}(x)\right\}
$$

Since $\underline{v}(t, x, \psi(x)) \leqslant \bar{v}(t, x, \psi(x))$ for all $(t, x) \in \mathbb{R}^{2}$ and since $\underline{v}$ and $\bar{v}$ are globally Lipschitzcontinuous in $\mathbb{R} \times \bar{\Omega}$, it follows that

$$
\underline{v}_{\infty}\left(t, x, \psi_{\infty}(x)\right) \leqslant \bar{v}_{\infty}\left(t, x, \psi_{\infty}(x)\right)
$$

for all $(t, x) \in \mathbb{R}^{2}$.

By passage to the limit, the functions $\underline{v}_{\infty}$ and $\bar{v}_{\infty}$ satisfy

$$
\begin{cases}\left(\underline{v}_{\infty}\right)_{t} \leqslant \Delta \underline{v}_{\infty}+\kappa \partial_{y} \underline{v}_{\infty}+g\left(\underline{v}_{\infty}\right) & \text { in } \mathcal{D}^{\prime}\left(\mathbb{R} \times \Omega_{\infty}\right) \\ \left(\bar{v}_{\infty}\right)_{t} \geqslant \Delta \bar{v}_{\infty}+\kappa \partial_{y} \bar{v}_{\infty}+g\left(\bar{v}_{\infty}\right) & \text { in } \mathcal{D}^{\prime}\left(\mathbb{R} \times \Omega_{\infty}\right)\end{cases}
$$

and $\underline{v}_{\infty}-\varepsilon^{*} \leqslant \underline{v}_{\infty}$ in $\mathbb{R} \times \overline{\Omega_{\infty}}$. On the other hand, $\psi_{\infty}(0) \geqslant A>0$ from (3.1), and

$$
\underline{v}_{\infty}(0,0,0)-\varepsilon^{*}=\underline{v}_{\infty}(0,0,0) .
$$

Lastly, $\underline{v}_{\infty}-\varepsilon^{*} \leqslant \underline{v}_{\infty} \leqslant \delta$ in $\mathbb{R} \times \overline{\Omega_{\infty}}$ and the function $g$ was assumed to be nonincreasing in $(-\infty, \delta]$. Hence, $g\left(\underline{v}_{\infty}-\varepsilon^{*}\right) \geqslant g\left(\underline{v}_{\infty}\right)$ in $\mathbb{R} \times \overline{\Omega_{\infty}}$.

Therefore, the function $w:=\underline{v}_{\infty}-\varepsilon^{*}-\bar{v}_{\infty}$ is a bounded, globally Lipschitz-continuous and nonpositive function in $\mathbb{R} \times \overline{\Omega_{\infty}}$, vanishing at the point $(0,0,0)$ and satisfying

$$
w_{t} \leqslant \Delta w+\kappa \partial_{y} w+\gamma(t, x, y) w \quad \text { in } \mathcal{D}^{\prime}\left(\mathbb{R} \times \Omega_{\infty}\right),
$$

where $\gamma$ is globally bounded function (here we use the fact that $g$ is globally Lipschitzcontinuous). The strong parabolic maximum principle then implies that $w(t, x, y)=0$, i.e. $\underline{v}_{\infty}(t, x, y)-\varepsilon^{*}=\bar{v}_{\infty}(t, x, y)$, for all $t \leqslant 0$ and $(x, y) \in \overline{\Omega_{\infty}}$. But the positivity of $\varepsilon^{*}$ contradicts the fact that $\underline{v}_{\infty} \leqslant \bar{v}_{\infty}$ on $\mathbb{R} \times \partial \Omega_{\infty}$.

As a conclusion, $\varepsilon^{*}=0$ and $\underline{v} \leqslant \bar{v}$ in $\mathbb{R} \times \bar{\Omega}$.

Remark 3.2. - The above comparison principle is a version of a parabolic maximum principle for time-global solutions in an unbounded space-domain. This comparison principle actually holds the same way in any space-dimension for more general second-order parabolic operators with smooth coefficients depending on time and space and a non-linearity $g\left(t, x_{1}, \ldots, x_{N}, u\right)$ satisfying the same monotonicity assumption with respect to $u$ as in Proposition 3.1.

Let us now turn to the

Proof of Theorem 1.3. - Under the assumptions of Theorem 1.3, the function $v$ defined in $\mathbb{R}^{3}$ by $v(t, x, y):=u(t, x, y-c t)$ is such that $0 \leqslant v \leqslant 1$ and it solves

$$
v_{t}=\Delta v-c \partial_{y} v+f(v) .
$$


From standard parabolic estimates, the function $v$ is globally Lipschitz-continuous with respect to all variables $(t, x, y)$. Furthermore, $v$ satisfies

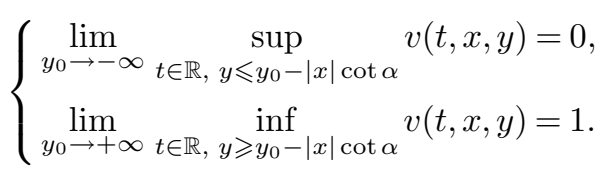

We shall now prove that $v$ is actually independent of $t$. That will imply that $v=v(x, y)$ is a solution of (1.1)-(1.2) (notice that from the strong maximum principle, one then has $0<v<1$ ). From Theorem 1.1 and from the uniqueness results in [19], it will follow that $v=\tau_{a, b} \phi$ in $\mathbb{R}^{2}$, for some pair $(a, b) \in \mathbb{R}^{2}$.

Fix now any real number $t_{0}$. For $s \in \mathbb{R}$, call $w^{s}$ the function defined in $\mathbb{R}^{3}$ by

$$
w^{s}(t, x, y)=v\left(t+t_{0}, x, y+s\right) .
$$

The function $w^{s}$ is a solution of (3.2) as well.

From the assumptions on $f$, there exists $\rho>0$ such that $\theta \leqslant 1-\rho$ and $f$ is nonincreasing on the interval $[1-\rho,+\infty)$. Remember also that $f$ is identically equal to 0 on $(-\infty, \theta]$. From (3.3), there exists $A>0$ such that

$$
\begin{cases}v(t, x, y) \geqslant 1-\rho & \text { for all } t \in \mathbb{R}, y \geqslant A-|x| \cot \alpha, \\ v(t, x, y) \leqslant \theta & \text { for all } t \in \mathbb{R}, y \leqslant-A-|x| \cot \alpha .\end{cases}
$$

Choose any $s \geqslant 2 A$ and observe that

$$
\begin{aligned}
w^{s}(t, x,-A-|x| \cot \alpha) & =v\left(t+t_{0}, x, s-A-|x| \cot \alpha\right) \\
& \geqslant 1-\rho \geqslant \theta \geqslant v(t, x,-A-|x| \cot \alpha)
\end{aligned}
$$

for all $(t, x) \in \mathbb{R}^{2}$. It is then immediate to check that all the assumptions of Proposition 3.1 are satisfied with $g=f, \delta=\theta, \psi(x)=-A-|x| \cot \alpha, \kappa=-c, \underline{v}=v, \bar{v}=w^{s}$. Therefore,

$$
w^{s}(t, x, y) \geqslant v(t, x, y) \quad \text { for all } t \in \mathbb{R} \text { and } y \leqslant-A-|x| \cot \alpha .
$$

Similarly, the assumptions of Proposition 3.1 are also satisfied with the choices

$$
\begin{aligned}
& g(\tau)=-f(1-\tau), \quad \delta=\rho, \quad \psi(x)=A+|x| \cot \alpha, \quad \kappa=c, \\
& \underline{v}(t, x, y)=1-w^{s}(t, x,-y) \quad \text { and } \quad \bar{v}(t, x, y)=1-v(t, x,-y) .
\end{aligned}
$$

Therefore, $\underline{v}(t, x, y) \leqslant \bar{v}(t, x, y)$ for all $t \in \mathbb{R}$ and $y \leqslant A+|x| \cot \alpha$, which means that

$$
v(t, x, y) \leqslant w^{s}(t, x, y) \quad \text { for all } t \in \mathbb{R} \text { and } y \geqslant-A-|x| \cot \alpha .
$$

As a consequence, one has $v \leqslant w^{s}$ in $\mathbb{R}^{3}$ for all $s \geqslant 2 A$. Let us now define

$$
s^{*}=\inf \left\{s>0, v \leqslant w^{\tau} \text { in } \mathbb{R}^{3} \text { for all } \tau \geqslant s\right\} .
$$

By continuity, one has $v \leqslant w^{s^{*}}$. Let us assume by contradiction that $s^{*}>0$. One shall consider two cases, namely whether the infimum of $w^{s^{*}}-v$ is positive or zero on the strip

$$
S=\left\{(t, x, y) \in \mathbb{R}^{3},|y+| x|\cot \alpha| \leqslant A\right\} .
$$


Case 1: $\inf _{S}\left(w^{s^{*}}-v\right)>0$. Since the function $v$, as well as $w^{s^{*}}$, is globally Lipschitzcontinuous, there exists $\eta_{0} \in\left(0, s^{*}\right)$ such that $w^{s^{*}-\eta} \geqslant v$ in $S$ for all $\eta \in\left[0, \eta_{0}\right]$. Choose any $\eta \in\left[0, \eta_{0}\right]$. Since $s^{*}-\eta \geqslant 0$, one has $w^{s^{*}-\eta}(t, x, y) \geqslant 1-\rho$ for all $t \in \mathbb{R}$ and $y \geqslant A-|x| \cot \alpha$. It also follows from the choice of $\eta$ that

$$
w^{s^{*}-\eta}(t, x, A-|x| \cot \alpha) \geqslant v(t, x, A-|x| \cot \alpha)
$$

for all $(t, x) \in \mathbb{R}^{2}$ (since $(t, x, A-|x| \cot \alpha) \in \partial S$ for all $(t, x) \in \mathbb{R}^{2}$ ). As above, it is straightforward to check that Proposition 3.1 implies that

$$
w^{s^{*}-\eta}(t, x, y) \geqslant v(t, x, y) \quad \text { for all } t \in \mathbb{R} \text { and } y \geqslant A-|x| \cot \alpha .
$$

Similarly, it can also be deduced that

$$
w^{s^{*}-\eta}(t, x, y) \geqslant v(t, x, y) \quad \text { for all } t \in \mathbb{R} \text { and } y \leqslant-A-|x| \cot \alpha .
$$

Putting all the preceding facts together, one concludes that $w^{s^{*}-\eta} \geqslant v$ in $\mathbb{R}^{3}$ for all $\eta \in\left[0, \eta_{0}\right]$. This is in contradiction with the minimality of $s^{*}$, since $\eta_{0}>0$. Therefore, case 1 is ruled out.

Case 2: $\inf _{S}\left(w^{s^{*}}-v\right)=0$. There exists then a sequence $\left(t_{n}, x_{n}, y_{n}\right)$ such that $t_{n} \in \mathbb{R}$, $-A-\left|x_{n}\right| \cot \alpha \leqslant y_{n} \leqslant A-\left|x_{n}\right| \cot \alpha$ and

$$
w^{s^{*}}\left(t_{n}, x_{n}, y_{n}\right)-v\left(t_{n}, x_{n}, y_{n}\right) \rightarrow 0 \quad \text { as } n \rightarrow+\infty
$$

Call $v_{n}(t, x, y)=v\left(t+t_{n}, x+x_{n}, y+y_{n}\right)$. Each function $v_{n}$ is a solution of (3.2) and ranges in $[0,1]$. From standard parabolic estimates, the functions $v_{n}$ converge locally uniformly, up to extraction of some subsequence, to a global solution $v_{\infty}$ of (3.2) such that $0 \leqslant v_{\infty} \leqslant 1$. Furthermore, $v_{\infty}\left(t_{0}, 0, s^{*}\right)=v_{\infty}(0,0,0)$. Therefore, the function

$$
z(t, x, y):=v_{\infty}\left(t+t_{0}, x, y+s^{*}\right)-v_{\infty}(t, x, y)
$$

which is nonnegative since $v \leqslant w^{s^{*}}$, vanishes at $(0,0,0)$ and is a global bounded solution of

$$
z_{t}=\Delta z-c \partial_{y} z+\gamma(t, x, y) z
$$

for some bounded function $\gamma$ (here we use the fact that $f$ is globally Lipschitz-continuous). The strong maximum principle for $t \leqslant 0$ and the uniqueness of the Cauchy problem for the above equation then imply that $z(t, x, y)=0$ for all $(t, x, y) \in \mathbb{R}^{3}$. As a consequence,

$$
v_{\infty}\left(t+n t_{0}, x, y+n s^{*}\right)=v_{\infty}(t, x, y)
$$

for all $(t, x, y) \in \mathbb{R}^{3}$ and $n \in \mathbf{Z}$.

Furthermore, from the definitions of $\left(t_{n}, x_{n}, y_{n}\right)$, one of the following three cases occur up to extraction of some subsequence: (i) the sequence $\left(x_{n}, y_{n}\right)$ is bounded, (ii) $x_{n} \rightarrow-\infty$, or (iii) $x_{n} \rightarrow+\infty$.

If case (i) occurs, then (3.3) holds for $v_{\infty}$. If case (ii) occurs, then the function $v_{\infty}$ satisfies

$$
\left\{\begin{array}{l}
\lim _{y_{0} \rightarrow-\infty} \sup _{t \in \mathbb{R},} v_{y \leqslant y_{0}+x \cot \alpha} v_{\infty}(t, x, y)=0, \\
\lim _{y_{0} \rightarrow+\infty} \inf _{t \in \mathbb{R},} v_{y \geqslant y_{0}+x \cot \alpha}(t, x, y)=1 .
\end{array}\right.
$$


Lastly, if case (iii) occurs, then $v_{\infty}$ satisfies

$$
\left\{\begin{array}{l}
\lim _{y_{0} \rightarrow-\infty} \sup _{t \in \mathbb{R},} v_{\infty \leqslant y_{0}-x \cot \alpha}(t, x, y)=0, \\
\lim _{y_{0} \rightarrow+\infty} \inf _{t \in \mathbb{R},} v_{y \geqslant y_{0}-x \cot \alpha}(t, x, y)=1 .
\end{array}\right.
$$

In each of the three cases (i), (ii) or (iii), one gets a contradiction with property (3.4). Therefore case 2 is ruled out too.

As a conclusion, the assumption $s^{*}>0$ is impossible, whence

$$
v(t, x, y) \leqslant w^{0}(t, x, y)=v\left(t+t_{0}, x, y\right)
$$

for all $(t, x, y) \in \mathbb{R}^{3}$. Since $t_{0}$ is arbitrary in $\mathbb{R}$, one concludes that $v$ does not depend on the variable $t$. As already emphasized, that completes the proof of Theorem 1.3.

Let us now turn to the

Proof of Theorem 1.5. - The functions $v_{n}(t, x, y)=u\left(t_{n}+t, x, y-c t_{n}-c t\right)$ solve

$$
\partial_{t} v_{n}=\Delta v_{n}-c \partial_{y} v_{n}+f\left(v_{n}\right)
$$

for $t>-t_{n}$. Furthermore, since $\phi$ is a solution of (1.1), the maximum principle implies that

$$
v_{n}(t, x, y) \leqslant \phi(x, y)
$$

for all $(x, y) \in \mathbb{R}^{2}$ and for all $t \geqslant-t_{n}$. On the other hand, because of the second inequality in (1.8) and because $u_{0}$ is nonnegative, there exist $\eta \in(\theta, 1]$ and $s_{0} \in \mathbb{R}$ such that

$$
\forall(x, y) \in \mathbb{R}^{2}, \quad u_{0}(x, y) \geqslant \max \left(H\left( \pm x \cos \alpha+y \sin \alpha+s_{0}\right)\right),
$$

where $H(s)=0$ if $s<0$ and $H(s)=\eta$ if $s \geqslant 0$. Therefore,

$$
\forall t \geqslant-t_{n}, \forall(x, y) \in \mathbb{R}^{2}, \quad v_{n}(t, x, y) \geqslant \max \left(v^{+}\left(t_{n}+t, x, y\right), v^{-}\left(t_{n}+t, x, y\right)\right),
$$

where the functions $v^{ \pm}$solve Eq. (3.5) with initial conditions

$$
v^{ \pm}(0, x, y)=H\left( \pm x \cos \alpha+y \sin \alpha+s_{0}\right) .
$$

Consider the function $v^{+}$. Since Eq. (3.5) is invariant up to translation and since $v^{+}(0, \cdot, \cdot)$ only depends on the variable $s=x \cos \alpha+y \sin \alpha$, so does $v^{+}(t, \cdot, \cdot)$ at any time $t \geqslant 0$. Therefore, $v^{+}(t, x, y)$ can be written as $v^{+}(t, x, y)=V^{+}(t, s)$ where $V^{+}$solves

$$
\left\{\begin{array}{l}
\partial_{t} V^{+}=\partial_{s}^{2} V^{+}-c_{0} \partial_{s} V^{+}+f\left(V^{+}\right) \\
V^{+}(0, s)=H\left(s+s_{0}\right)
\end{array}\right.
$$

A result of Kanel' [25,26] (see also Roquejoffre [35]) yields the convergence of $V^{+}(t, s)$ to $\phi_{0}\left(s+s_{1}\right)$ uniformly in $s \in \mathbb{R}$ as $t \rightarrow+\infty$, for some $s_{1} \in \mathbb{R}$, where $\phi_{0}$ is the solution of (1.5) such that, say, $\phi_{0}(0)=\theta$. By symmetry in the $x$-variable, it follows that $v^{-}(t, x, y) \rightarrow \phi_{0}\left(s^{\prime}+s_{1}\right)$ uniformly in $(x, y) \in \mathbb{R}^{2}$ as $t \rightarrow+\infty$, where $s^{\prime}=-x \cos \alpha+y \sin \alpha$. Consequently,

$$
\forall(t, x, y) \in \mathbb{R}^{3}, \quad \liminf _{n \rightarrow+\infty} v_{n}(t, x, y) \geqslant \max \left(\phi_{0}\left( \pm x \cos \alpha+y \sin \alpha+s_{1}\right)\right) .
$$


Eventually, from standard parabolic estimates, there exists a subsequence $n^{\prime} \rightarrow+\infty$ such that the functions $v_{n^{\prime}}$ converge locally uniformly in $\mathbb{R} \times \mathbb{R}^{2}$ to a classical solution $v(t, x, y)$ of $v_{t}=\Delta v-c v_{y}+f(v)$ such that

$$
\max \left(\phi_{0}\left( \pm x \cos \alpha+y \sin \alpha+s_{1}\right)\right) \leqslant v(t, x, y) \leqslant \phi(x, y)
$$

for all $(t, x, y) \in \mathbb{R}^{3}$.

The function $u(t, x, y)=v(t, x, y+c t)$ then satisfies (1.6) and (1.7). Theorem 1.3 yields that $u(t, x, y)=\phi(x+a, y+b+c t)$ for some $(a, b) \in \mathbb{R}^{2}$ and for all $(t, x, y) \in \mathbb{R} \times \mathbb{R}^{2}$. Therefore, $v(t, x, y)=\phi(x+a, y+b)$ and the conclusion of Theorem 1.5 follows.

\section{Local stability}

The goal of this section is to prove the following stability result:

THEOREM 4.1 (Local stability). - Let $\alpha \in(0, \pi / 2)$ and $f$ satisfy (1.4). Let $u(t, x, y)$ be a solution of the Cauchy problem (1.3). There exists $\bar{\rho}>0$ (one may choose $\bar{\rho}=c_{0} \cot \alpha$ ) such that the following holds: for any $\rho \in(0, \bar{\rho})$, there is $\varepsilon>0$ such that if $0 \leqslant u_{0} \leqslant 1, u_{0} \in U C\left(\mathbb{R}^{2}\right)$, and $\left\|u_{0}-\phi\right\|_{G_{\rho}} \leqslant \varepsilon$ for some solution $\phi$ of (1.1)-(1.2), then there are two constants $K \geqslant 0$ and $\omega>0$ such that

$$
\forall t \geqslant 0, \quad\|u(t, \cdot, \cdot-c t)-\phi(\cdot, \cdot)\|_{G_{\rho}} \leqslant K e^{-\omega t} .
$$

The object to study is the linearized operator around a wave solution $\phi$ :

$$
L v=-\Delta v+c \partial_{y} v-f^{\prime}(\phi) v .
$$

In the whole section, we choose the (unique) wave $\phi$ solving (1.1)-(1.2) such that:

$$
\phi(x, y)=\phi(-x, y), \quad \phi(0,0)=\theta .
$$

Proposition 4.2 (No eigenvalue with negative real part). - Let $\rho>0$ and $u \in C\left(\mathbb{R}^{2}, \mathbf{C}\right)$ be a classical solution of $L u=\lambda u$ such that $\operatorname{Re}(u), \operatorname{Im}(u) \in G_{\rho}$.

- If $\operatorname{Re}(\lambda)<0$, then $u=0$.

- If $\operatorname{Re}(\lambda)=0$, then there is $C>0$ such that

$$
|u| \leqslant C \phi_{y} \quad \text { in } \mathbb{R}^{2} .
$$

Proof. - We wish to follow the idea in [5]. The result is obtained by proving first that $u$ decays faster than any derivative of the wave, then to conclude with the aid of the parabolic equation

$$
U_{t}+L U=0, \quad U(0)=\operatorname{Re}(u)
$$

This first part of the programme does not seem to be done as easily as in [5], due to the lack of precise boundaries where to apply an exact boundary condition - hence an evolution equation approach.

In order to circumvent the difficulty we directly use Eq. (4.1) and construct a Fife-McLeod type super-solution (see [17]): set

$$
w_{0}(x, y)=\min \left(e^{\tilde{\rho}(y \sin \alpha-x \cos \alpha)}, e^{\tilde{\rho}(y \sin \alpha+x \cos \alpha)}\right),
$$

where $\tilde{\rho} \in\left(0, c_{0}\right)$ shall be chosen later. We also set 


$$
\bar{U}(t, x, y)=a_{0}(t) \phi_{y}(x, y)+a_{1}(t) \gamma_{1}(x, y)+a_{2}(t) w_{0}(x, y) \gamma_{2}(x, y) .
$$

Define $y_{1}>0$ and $k>0$ such that

$$
\forall(x, y) \in \mathcal{C}_{+}\left(y_{1}, \pi-\alpha\right), \quad f^{\prime}(\phi(x, y)) \leqslant-k
$$

and choose $y_{2}<0$ such that

$$
\forall(x, y) \in \mathcal{C}_{-}\left(y_{2}, \alpha\right), \quad f^{\prime}(\phi(x, y))=0 .
$$

Actually, any negative $y_{2}$ works since $\phi(0,0)=\theta$ and it is known $([9,19])$ that $\phi$ is nonincreasing in any direction $\tau=(\cos \beta, \sin \beta)$ such that $-\pi / 2-\alpha \leqslant \beta \leqslant-\pi / 2+\alpha$. The functions $\gamma_{1}$ and $\gamma_{2}$ are required to be in $C^{2}\left(\mathbb{R}^{2}\right)$ and to satisfy

- $0 \leqslant \gamma_{1}, \gamma_{2} \leqslant 1$ in $\mathbb{R}^{2}$;

- $\gamma_{1} \equiv 1$ in $\mathcal{C}_{+}\left(2 y_{1}, \pi-\alpha\right)$ and $\gamma_{1} \equiv 0$ in $\mathcal{C}_{-}\left(y_{1}, \alpha\right)$;

- $\gamma_{2} \equiv 1$ in $\mathcal{C}_{-}\left(2 y_{2}, \alpha\right)$ and $\gamma_{2} \equiv 0$ in $\mathcal{C}_{+}\left(y_{2}, \pi-\alpha\right)$;

- $\partial_{x} \gamma_{1}(0, y)=\partial_{x} \gamma_{2}(0, y) \equiv 0$.

Then set

$$
\mathcal{L} \bar{U}=\bar{U}_{t}+L \bar{U}
$$

as is now classical we anticipate that $\dot{a}_{0}(t)$ will be nonnegative, and break the evaluation of $\mathcal{L} \bar{U}$ in three parts.

1. $(x, y) \in \mathcal{C}_{-}\left(2 y_{2}, \alpha\right)$. Then we have, because of (4.3) and because $\phi_{y} \geqslant 0$ :

$$
\mathcal{L} \bar{U} \geqslant\left(\dot{a}_{2}(t)+\left(c \tilde{\rho} \sin \alpha-\tilde{\rho}^{2}\right) a_{2}(t)\right) w_{0},
$$

provided that $a_{2}(t)$ is nonnegative. Remember that $c \sin \alpha=c_{0}$ and set

$$
a_{2}(t)=\alpha_{2} e^{-t \tilde{\rho}\left(c_{0}-\tilde{\rho}\right)}
$$

with $\alpha_{2}>0$ to be chosen later. Observe here that $\tilde{\rho}\left(c_{0}-\tilde{\rho}\right)>0$ since $\tilde{\rho}$ is in $\left(0, c_{0}\right)$.

2. $(x, y) \in \mathcal{C}_{+}\left(2 y_{1}, \pi-\alpha\right)$. Then we have, because of (4.2) and provided that $a_{1}(t)$ is nonnegative:

$$
\mathcal{L} \bar{U} \geqslant \dot{a}_{1}(t)+k a_{1}(t)
$$

and we define

$$
a_{1}(t)=\alpha_{1} e^{-k t}
$$

with $\alpha_{1}>0$ to be chosen later.

3. $(x, y) \in \mathcal{C}_{-}\left(2 y_{1}, \alpha\right) \cap \mathcal{C}_{+}\left(2 y_{2}, \pi-\alpha\right)$. There is a large constant $C_{1}>0$ and a small positive constant $\omega$ such that

$$
\mathcal{L} \bar{U} \geqslant \dot{a}_{0}(t) \phi_{y}(x, y)-C_{1} e^{-\omega t} .
$$

Then, because $\phi_{y}$ is positive and bounded away from 0 in the region under consideration, there is a large constant $C_{2}$ such that we may take

$$
a_{0}(t)=\alpha_{0}-C_{2} e^{-\omega t}, \quad \alpha_{0}>0,
$$

and

$$
\mathcal{L} \bar{U} \geqslant 0 \quad \text { in } \mathcal{C}_{-}\left(2 y_{1}, \alpha\right) \cap \mathcal{C}_{+}\left(2 y_{2}, \pi-\alpha\right) .
$$


Combining the above steps, and since $\operatorname{Re}(u) \in G_{\rho}$, one can choose $\alpha_{0}, \alpha_{1}, \alpha_{2}$ large enough and $\tilde{\rho}>0$ small enough so that $\bar{U}$ satisfies $\mathcal{L} \bar{U} \geqslant 0$ in $\mathbb{R}_{+} \times \mathbb{R}^{2}$ and $\operatorname{Re}(u) \leqslant \bar{U}(0,$.$) . Then we$ have:

$$
\operatorname{Re}\left(e^{-\lambda t} u\right)(t, x, y) \leqslant \bar{U}(t, x, y) .
$$

We may repeat the argument with $-\operatorname{Re}(u)$ so as to obtain a similar lower bound for $\operatorname{Re}\left(e^{-\lambda t} u\right)$.

We can now conclude:

- If $\operatorname{Re}(\lambda)<0$, assuming $u \neq 0$ contradicts the unboundedness of $\operatorname{Re}\left(e^{-\lambda t} u\right)$.

- If $\operatorname{Re}(\lambda)=0$, then we argue similarly with $\operatorname{Im}(u)$ and $\operatorname{Im}\left(e^{-\lambda t} u\right)$ and we get an upper bound of the type $\left|\operatorname{Im}\left(e^{-\lambda t} u\right)\right| \leqslant \bar{V}(t, x, y)$, where $\bar{V}$ is of the same type as $\bar{U}$. We then only have to let $t \rightarrow+\infty$ to get that $|u| \leqslant C \phi_{y}$ in $\mathbb{R}^{2}$.

This ends the proof of Proposition 4.2.

The next step is to show that 0 is NOT an eigenvalue of $L$ when $L$ is restricted to $G_{\rho}$. We first observe that

$$
L\left(\phi_{x}\right)=L\left(\phi_{y}\right)=0,
$$

but neither $\phi_{x}$ nor $\phi_{y}$ belongs to $G_{\rho}$ since

$$
\liminf _{|x| \rightarrow+\infty} \phi_{x}(x,-|x| \cot \alpha) \quad \text { and } \quad \liminf _{|x| \rightarrow+\infty} \phi_{y}(x,-|x| \cot \alpha)
$$

are positive (indeed, $\phi(x+\xi, y-|\xi| \cot \alpha) \rightarrow \phi_{0}\left( \pm x \cos \alpha+y \sin \alpha+t_{ \pm}\right)$as $\xi \rightarrow \pm \infty$ in $C_{\text {loc }}^{2}\left(\mathbb{R}^{2}\right)$, for some $\left.t_{ \pm} \in \mathbb{R}\right) .^{3}$

Then remark that a function $u(x, y)$ may be decomposed in an even and odd part (with respect to $x): u=u_{1}+u_{2}$ with

$$
u_{1}(x, y)=\frac{u(x, y)+u(-x, y)}{2}, \quad u_{2}(x, y)=\frac{u(x, y)-u(-x, y)}{2} .
$$

Notice also that $\partial_{x} u_{1}(0, y)=0$ - provided $u_{1}$ is smooth enough - and that $u_{2}(0, y)=0$. This trivial remark implies in fact boundary conditions for $u_{1}$ and $u_{2}$ on the $y$-axis if $u_{1}$ and $u_{2}$ are considered as functions from the right half-space that we denote $\mathbb{R}_{+}^{2}=\{x>0\}$. Notice finally that $\phi_{x}$ is odd and $\phi_{y}$ is even (with respect to the $x$-variable).

On the other hand, the operator $L$ commutes with the reflections with respect to the $y$ axis. Hence, if a function $u$ in $G_{\rho}$ solves $L u=0$, then both functions $u_{1}$ and $u_{2}$ are in $G_{\rho}$ and solve $L u=0$. On the basis of all the above remarks we have the

Proposition 4.3 (No eigenfunctions in the null space of $L$ ). - (i) Let $\rho>0$ and $u \in$ $C^{2}\left(\overline{\mathbb{R}_{+}^{2}}\right) \cap G_{\rho}$ solve

$$
L u=0 \quad \text { in } \mathbb{R}_{+}^{2}, \quad u(0, y)=0 .
$$

Then $u=0$.

(ii) Let $\rho>0$ and $u \in C^{2}\left(\overline{\mathbb{R}_{+}^{2}}\right) \cap G_{\rho}$ solve

$$
L u=0 \quad \text { in } \mathbb{R}_{+}^{2}, \quad u_{x}(0, y)=0 .
$$

Then $u=0$.

\footnotetext{
${ }^{3}$ We have of course $t_{-}=t_{+}$as soon as $\phi$ is symmetric with respect to the $x$-variable.
} 
We will only prove part (i) of Proposition 4.3, part (ii) being completely similar and being actually included in the proof of Proposition 4.5 below. The proof of part (i) will be based on the following

LEMMA 4.4. - Let $\rho>0$ and $u \in C^{2}\left(\overline{\mathbb{R}_{+}^{2}}\right) \cap G_{\rho}$ satisfy (4.4). Then there is a constant $C>0$ such that

$$
|u| \leqslant C \phi_{x} \quad \text { in } \mathbb{R}_{+}^{2} .
$$

Proof. - Argue as in Proposition 4.2, but this time $u$ vanishes at the boundary $\{x=0\}$, as well as $\phi_{x}$. To circumvent this we define the supersolution $\bar{U}$ as

$$
\bar{U}(t, x, y)=a_{0}(t) \phi_{x}(x, y)+\left(a_{1}(t) \gamma_{1}(x, y)+a_{2}(t) w_{0}(x, y) \gamma_{2}(x, y)\right) w_{1}(x)
$$

where $w_{1}$ is bounded, increasing and concave, and satisfies moreover $w_{1}(0)=w_{1}^{\prime \prime}(0)=0$ and $w_{1}(+\infty)=1$. With similar choices for the functions $a_{0}, a_{1}, a_{2}, w_{0}$ as in Proposition 4.2, one has $\mathcal{L} \bar{U} \geqslant 0$ for all $t \geqslant 0$ and $(x, y) \in \mathbb{R}_{+}^{2}$. Furthermore, since $L \phi_{x}=0, \phi_{x}>0$ in $\mathbb{R}_{+}^{2}$ and $\phi_{x}=0$ on $\{x=0\}$, it follows from Hopf lemma that $\phi_{x x}(0, y)>0$ for all $y \in \mathbb{R}$. On the other hand, the standard elliptic estimates up to the boundary imply that, say,

$$
\|\nabla u\|_{L^{\infty}\left(\left\{0 \leqslant x \leqslant 1, y_{0} \leqslant y \leqslant y_{0}+1\right\}\right)} \leqslant C_{0}\|u\|_{L^{\infty}\left(\left\{0 \leqslant x \leqslant 2, y_{0}-1 \leqslant y \leqslant y_{0}+2\right\}\right)}
$$

for some constant $C_{0}$ independent of $y_{0} \in \mathbb{R}$. Therefore, suitable choices of $a_{0}, a_{1}, a_{2}$ and $\tilde{\rho}$ guarantee that $u(x, y) \leqslant \bar{U}(0, x, y)$ in $\mathbb{R}_{+}^{2}$. Hence, $u(x, y) \leqslant \bar{U}(t, x, y)$ for all $t \geqslant 0$ and $(x, y) \in \mathbb{R}_{+}^{2}$. Passing to the limit $t \rightarrow+\infty$ as in Proposition 4.2 leads to $u \leqslant C \phi_{x}$ in $\mathbb{R}_{+}^{2}$.

The same reasoning with $-u$ completes the proof of Lemma 4.4.

Proof of Proposition 4.3. - As already emphasized, we will only prove part (i). Under the assumptions of part (i), and from Lemma 4.4, let us denote by $C_{0}$ the biggest (maybe negative) constant $C$ such that $u \geqslant C \phi_{x}$ in $\mathbb{R}_{+}^{2}$. We would like to prove that $C_{0} \geqslant 0$. To see this, we assume the contrary and try to prove that $u \geqslant\left(C_{0}+\delta\right) \phi_{x}$, for all $\delta$ in a small range.

First of all, since $u \in G_{\rho}, \phi_{x} \notin G_{\rho}$ and $C_{0} \neq 0$, one gets that $u \neq C_{0} \phi_{x}$. Therefore, $u>C_{0} \phi_{x}$ in $\mathbb{R}_{+}^{2}$ and $u_{x}(0, y)>C_{0} \phi_{x x}(0, y)$ for all $y \in \mathbb{R}$, due to the strong maximum principle and the Hopf lemma. Consequently, for all subdomain $\Omega$ of $\mathbb{R}_{+}^{2}$ such that $\mathbb{R}_{+}^{2} \backslash \Omega$ is bounded, there exists $\delta_{0}(\Omega)>0$ such that

$$
\forall \delta \in\left[0, \delta_{0}(\Omega)\right], \forall(x, y) \in \mathbb{R}_{+}^{2} \backslash \Omega, \quad u(x, y) \geqslant\left(C_{0}+\delta\right) \phi_{x}(x, y) .
$$
let

Rotate the coordinates $(x, y)$ so as to bring the vector $(1,0)$ to the vector $e_{\alpha}$ defined by $(1.10)$;

$$
(X, Y)=(x \sin \alpha-y \cos \alpha, x \cos \alpha+y \sin \alpha)
$$

be the new coordinates.

In this new system the operator $L$ reads

$$
L=-\Delta-c \cos \alpha \frac{\partial}{\partial X}+c_{0} \frac{\partial}{\partial Y}-f^{\prime}(\phi) .
$$

To describe some portions of the plane, we will indifferently use the $(x, y)$ or $(X, Y)$ coordinate system, and we make a slight abuse of notations identifying a point in $\Omega$ with its coordinates in the rotated frame. 


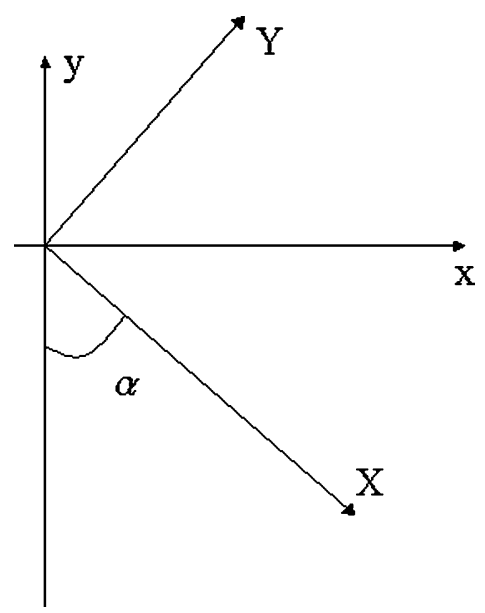

Fig. 2. Rotated axes.

Since $f^{\prime}\left(1^{-}\right)<0$ and $\phi \rightarrow 1^{-}$uniformly in $\mathcal{C}_{+}(y, \pi-\alpha)$ as $y \rightarrow+\infty$, one can then choose $Y_{1}>0$ such that:

$$
\exists k>0, \forall Y \geqslant Y_{1}, \quad f^{\prime}(\phi(X, Y)) \leqslant-k .
$$

Let $\Omega$ and $S$ be the subsets of $\mathbb{R}_{+}^{2}$ defined by

$$
\begin{gathered}
\Omega=\left\{x>0 \text { and }\left(Y>Y_{1} \text { or } X>1\right)\right\}, \\
S=\left\{X_{1} \geqslant 1,0 \leqslant Y \leqslant Y_{1}\right\},
\end{gathered}
$$

and let $\delta_{0}(\Omega)>0$ satisfy (4.5).

Since $u \geqslant C_{0} \phi_{x}$ in $\mathbb{R}_{+}^{2}$, two cases may occur:

Case 1: $\inf _{S}\left(u-C_{0} \phi_{x}\right)=0$. In that case, there exists a sequence $\left(X_{n}, Y_{n}\right)$ (in the $(X, Y)$ frame) such that $u\left(X_{n}, Y_{n}\right)-C_{0} \phi_{x}\left(X_{n}, Y_{n}\right) \rightarrow 0$ as $n \rightarrow+\infty$. Since the distance between $S$ and $\partial \mathbb{R}_{+}^{2}=\{x=0\}$ is positive and $u>C_{0} \phi_{x}$ in $\mathbb{R}_{+}^{2}$, one concludes that $X_{n} \rightarrow+\infty$. Furthermore, since the sequence $\left(Y_{n}\right)$ ranges in $\left[0, Y_{1}\right]$, one can assume, up to extraction of some subsequence, that $Y_{n} \rightarrow Y_{\infty}$ as $n \rightarrow+\infty$.

On the one hand, one has already mentioned the existence of $t_{+} \in \mathbb{R}$ such that

$$
\phi(x+\xi, y-|\xi| \cot \alpha) \rightarrow \phi_{0}\left(x \cos \alpha+y \sin \alpha+t_{+}\right)
$$

as $\xi \rightarrow+\infty$ in $C_{\text {loc }}^{2}\left(\mathbb{R}^{2}\right)$. Therefore, the functions $(X, Y) \mapsto \phi_{x}\left(X+X_{n}, Y+Y_{n}\right)$ locally converge to the function $\cos \alpha \phi_{0}^{\prime}\left(Y+Y_{\infty}+t_{+}\right)$as $n \rightarrow+\infty$.

On the other hand, from standard elliptic estimates, the functions

$$
(X, Y) \mapsto u_{n}\left(X+X_{n}, Y+Y_{n}\right)
$$

locally converge, up to extraction of some subsequence, to a solution $u_{\infty}(X, Y)$ of

$$
-\Delta u_{\infty}-c \cos \alpha \partial_{X} u_{\infty}+c_{0} \partial_{Y} u_{\infty}-f^{\prime}\left(\phi_{0}\left(Y+Y_{\infty}+t_{+}\right)\right) u_{\infty}=0 \quad \text { in } \mathbb{R}^{2} .
$$


Both functions $u_{\infty}$ and $C_{0} \cos \alpha \phi_{0}^{\prime}\left(Y+Y_{\infty}+t_{+}\right)$satisfy the above equation, and

$$
u_{\infty}(X, Y) \geqslant C_{0} \cos \alpha \phi_{0}^{\prime}\left(Y+Y_{\infty}+t_{+}\right) \quad \text { in } \mathbb{R}^{2}
$$

with equality at $(0,0)$. The strong maximum principle implies that

$$
u_{\infty}(X, Y) \equiv C_{0} \cos \alpha \phi_{0}^{\prime}\left(Y+Y_{\infty}+t_{+}\right) \quad \text { in } \mathbb{R}^{2} .
$$

But, since $u \in G_{\rho}$, one has, say, $u(X, 0) \rightarrow 0$ as $X \rightarrow+\infty$. Hence, $u_{\infty}\left(0,-Y_{\infty}\right)=0$, and $\phi_{0}^{\prime}\left(t_{+}\right)=0$ since $C_{0} \cos \alpha \neq 0$. But $\phi_{0}^{\prime}>0$ in $\mathbb{R}$. Therefore, case 1 is ruled out.

Case 2: $\inf _{S}\left(u-C_{0} \phi_{x}\right)>0$. In that case, since $\phi_{x}$ is globally bounded, there exists $\eta_{0}>0$ such that $u \geqslant\left(C_{0}+\eta\right) \phi_{x}$ in $S$ for all $\eta \in\left[0, \eta_{0}\right]$.

Choose now any $\delta$ such that $0<\delta \leqslant \min \left(\delta_{0}(\Omega), \eta_{0}\right)$. One then has $u \geqslant\left(C_{0}+\delta\right) \phi_{x}$ in $S \cup\left(\mathbb{R}_{+}^{2} \backslash \Omega\right)$. Let us now prove that the latter also holds in the two other parts of $\mathbb{R}_{+}^{2}$, namely in $\Omega_{1}=\{x>0, Y<0, X>1\}$ and $\Omega_{2}=\left\{x>0, Y>Y_{1}\right\}$.

Let us first deal with $\Omega_{1}$. Notice that $f(\phi)=f^{\prime}(\phi)=0$ in $\mathcal{C}_{-}(0, \alpha)$ since $\phi(0,0)=\theta$ and $\phi$ is nonincreasing in any direction of this cone $\mathcal{C}_{-}(0, \alpha)$. Hence, $f^{\prime}(\phi(X, Y))=0$ in $\Omega_{1}$ and both $u$ and $\left(C_{0}+\delta\right) \phi_{x}$ satisfy

$$
-\Delta v-c \cos \alpha v_{X}+c_{0} v_{Y}=0 \quad \text { in } \Omega_{1} .
$$

Furthermore, $u \geqslant\left(C_{0}+\delta\right) \phi_{x}$ on $\partial \Omega_{1}$. Lastly, remember that $|u| \leqslant C \phi_{x}$ in $\mathbb{R}^{2}$ from Lemma 4.4. Because of (1.2) and standard elliptic estimates, one can say that $\lim _{y \rightarrow-\infty} \sup _{\mathcal{C}_{-}(y, \alpha)}\left|\phi_{x}\right|=0$. Hence, as $\phi_{x}$ does, $u(X, Y) \rightarrow 0$ uniformly as $Y \rightarrow-\infty$ with $(X, Y) \in \Omega_{1}$. Therefore, with a method similar to the proof of Proposition 3.1 (see also Lemma 5.1 in [19]), one can prove that $u \geqslant\left(C_{0}+\delta\right) \phi_{x}-\varepsilon$ in $\Omega_{1}$ for all $\varepsilon>0$, whence $u \geqslant\left(C_{0}+\delta\right) \phi_{x}$ in $\Omega_{1}$.

Similarly, both $u$ and $\left(C_{0}+\delta\right) \phi_{x}$ satisfy

$$
-\Delta v-c \cos \alpha v_{X}+c_{0} v_{Y}-f^{\prime}(\phi) v=0 \quad \text { in } \Omega_{2},
$$

with $f^{\prime}(\phi(X, Y)) \leqslant 0$ in $\Omega_{2}$. Furthermore, $u \geqslant\left(C_{0}+\delta\right) \phi_{x}$ on $\partial \Omega_{2}$. Lastly,

$$
\lim _{y \rightarrow+\infty} \sup _{\mathcal{C}_{+}(y, \pi-\alpha)}\left|\phi_{x}\right|=0,
$$

whence $u(X, Y)$ and $\phi_{x}(X, Y) \rightarrow 0$ uniformly as $Y \rightarrow+\infty$ with $(X, Y) \in \Omega_{2}$. Since $\left(C_{0}+\delta\right) \phi_{x}-\varepsilon$ is a subsolution of (4.8) for all $\varepsilon>0$, it then follows similarly that

$$
u \geqslant\left(C_{0}+\delta\right) \phi_{x}-\varepsilon
$$

in $\Omega_{2}$ for all $\varepsilon>0$, whence $u \geqslant\left(C_{0}+\delta\right) \phi_{x}$ in $\Omega_{2}$.

As a conclusion, $u \geqslant\left(C_{0}+\delta\right) \phi_{x}$ in $\mathbb{R}_{+}^{2}$ for all $\delta \in\left[0, \min \left(\delta_{0}(\Omega), \eta_{0}\right)\right]$. This contradicts the definition of $C_{0}$.

Therefore, $C_{0} \geqslant 0$ and $u \geqslant 0$ in $\mathbb{R}_{+}^{2}$. However we would prove in the same way that $u \leqslant 0$ in $\mathbb{R}_{+}^{2}$. This proves $u=0$ in $\mathbb{R}_{+}^{2}$.

Proposition 4.5 (No eigenfunctions with pure imaginary eigenvalue).- Let $\rho>0$ and $u \in C^{2}\left(\mathbb{R}^{2}, \mathbf{C}\right)$ such that $\operatorname{Re}(u), \operatorname{Im}(u) \in G_{\rho}$. Assume that $L u=\lambda u$ with $\operatorname{Re}(\lambda)=0$ and $\operatorname{Im}(\lambda) \neq 0$. Then $u=0$.

Proof. - The proof is a generalisation of the above proposition, combined with the parabolic maximum principle. Once again, we may assume that $u$ is either odd or even; suppose it is 
even. If $u$ is as described above, Proposition 4.2 applies, and we may define the infimum (maybe nonpositive) of all $C$ such that $\operatorname{Re}(u) \leqslant C \phi_{y}$ in $\overline{\mathbb{R}_{+}^{2}}$ (or equivalently in $\mathbb{R}^{2}$ by evenness). Denote it by $C_{0}$.

We wish to prove that $C_{0} \leqslant 0$, as is now usual. Assume by contradiction that $C_{0}>0$.

Set $\lambda=i \omega$, with $\omega \neq 0$. The function

$$
U(t)=\operatorname{Re}\left(e^{-\lambda t} u\right)=\operatorname{Re}(u) \cos \omega t+\operatorname{Im}(u) \sin \omega t
$$

solves (4.1) in $\overline{\mathbb{R}_{+}^{2}}$ together with Neumann boundary conditions on $\partial \mathbb{R}_{+}^{2}$ for all $t \in \mathbb{R}$, as does $\phi_{y}$. Therefore, $U(t)(x, y) \leqslant C_{0} \phi_{y}(x, y)$ for all $(x, y) \in \overline{\mathbb{R}_{+}^{2}}$ and for all $t \geqslant 0$, whence for all $t \in \mathbb{R}$ since $U(t)$ is $2 \pi / \omega$-periodic in $t$.

If there exists $\left(x_{0}, y_{0}\right) \in \mathbb{R}_{+}^{2}$ such that $\operatorname{Re}(u)\left(x_{0}, y_{0}\right)=C_{0} \phi_{y}\left(x_{0}, y_{0}\right)$, then $U(t)-C_{0} \phi_{y}$ has an interior minimum at $t=0$ and $\left(x_{0}, y_{0}\right)$. Hence, $U(t) \equiv C_{0} \phi_{y}$ in $\mathbb{R}_{+}^{2}$ for all $t \leqslant 0$, and thus $\operatorname{Re}(u) \equiv C_{0} \phi_{y}$. The latter is impossible since $\operatorname{Re}(u) \in G_{\rho}$ and $\phi_{y} \notin G_{\rho}$. Therefore, $\operatorname{Re}(u)<C_{0} \phi_{y}$ in $\mathbb{R}_{+}^{2}$. Similarly, the parabolic Hopf lemma then implies that $\operatorname{Re}(u)<C_{0} \phi_{y}$ in $\partial \mathbb{R}_{+}^{2}$.

Under the notations in the proof of Proposition 4.3, let $S^{\prime}$ be the strip

$$
S^{\prime}=\left\{x \geqslant 0,0 \leqslant Y \leqslant Y_{1}\right\} .
$$

Since $\operatorname{Re}(u) \leqslant C_{0} \phi_{y}$ (in $\mathbb{R}^{2}$ ), two cases may occur:

Case 1: $\sup _{S^{\prime}}\left(\operatorname{Re}(u)-C_{0} \phi_{y}\right)=0$. Since $\operatorname{Re}(u)<C_{0} \phi_{y}$ in $\overline{\mathbb{R}_{+}^{2}}$, there exists then a sequence of points $\left(X_{n}, Y_{n}\right) \in S^{\prime}$ such that $X_{n} \rightarrow+\infty, Y_{n} \rightarrow Y_{\infty} \in \mathbb{R}$ and

$$
\operatorname{Re}(u)\left(X_{n}, Y_{n}\right)-C_{0} \phi_{y}\left(X_{n}, Y_{n}\right) \rightarrow 0 \quad \text { as } n \rightarrow+\infty .
$$

From standard elliptic estimates, the functions $(X, Y) \mapsto \operatorname{Re}(u)\left(X+X_{n}, Y+Y_{n}\right)$ and $(X, Y) \mapsto \operatorname{Im}(u)\left(X+X_{n}, Y+Y_{n}\right)$ converge, up to extraction of some subsequence, to two real-valued bounded functions $v_{\infty}(X, Y)$ and $w_{\infty}(X, Y)$ solving

$$
L_{\infty} v_{\infty}=-\omega w_{\infty} \text { and } L_{\infty} w_{\infty}=\omega v_{\infty} \quad \text { in } \mathbb{R}^{2},
$$

where

$$
L_{\infty}=-\Delta-c \cos \alpha \partial_{X}+c_{0} \partial_{Y}-f^{\prime}\left(\phi_{0}\left(Y+Y_{\infty}+t_{+}\right)\right) .
$$

Therefore, the function $u_{\infty}=v_{\infty}+i w_{\infty}$ solves $L_{\infty} u_{\infty}=\lambda u_{\infty}$.

On the other hand, one recalls that the functions $(X, Y) \mapsto \phi_{y}\left(X+X_{n}, Y+Y_{n}\right)$ locally converge to the function $\sin \alpha \phi_{0}^{\prime}\left(Y+Y_{\infty}+t_{+}\right)$as $n \rightarrow+\infty$.

Furthermore, $\operatorname{Re}\left(u_{\infty}\right)=v_{\infty} \leqslant C_{0} \sin \alpha \phi_{0}^{\prime}\left(Y+Y_{\infty}+t_{+}\right)$in $\mathbb{R}^{2}$ with equality at $(0,0)$, and both functions $\operatorname{Re}\left(e^{-\lambda t} u_{\infty}\right)$ and $C_{0} \sin \alpha \phi_{0}^{\prime}\left(Y+Y_{\infty}+t_{+}\right)$solve (4.1) with the operator $L_{\infty}$ instead of $L$. As done several lines above, one then concludes that

$$
v_{\infty}=\operatorname{Re}\left(u_{\infty}\right) \equiv C_{0} \sin \alpha \phi_{0}^{\prime}\left(Y+Y_{\infty}+t_{+}\right)
$$

But $\operatorname{Re}(u) \in G_{\rho}$, whence, say, $\operatorname{Re}(u)(X, 0) \rightarrow 0$ as $X \rightarrow+\infty$ and $v_{\infty}\left(0,-Y_{\infty}\right)=0$. Thus $\phi_{0}^{\prime}\left(t_{+}\right)=0$ since $C_{0} \sin \alpha \neq 0$. One gets a contradiction and case 1 is ruled out.

Case 2: $\sup _{S^{\prime}}\left(\operatorname{Re}(u)-C_{0} \phi_{y}\right)<0$. In that case, there exists $\eta_{0}>0$ such that

$$
\operatorname{Re}(u) \leqslant\left(C_{0}-\delta\right) \phi_{y}
$$


in $S^{\prime}$ for all $\delta \in\left[0, \eta_{0}\right]$. Choose any $\delta$ such that $0 \leqslant \delta \leqslant \eta_{0}$. Let $\Omega_{1}^{\prime}=\{x \geqslant 0, Y \leqslant 0\}$ and $\Omega_{2}^{\prime}=\left\{x \geqslant 0, Y \geqslant Y_{1}\right\}$, and let us prove that $\operatorname{Re}(u) \leqslant\left(C_{0}-\delta\right) \phi_{y}$ in $\Omega_{1}^{\prime} \cup \Omega_{2}^{\prime}$, which would yield that $\operatorname{Re}(u) \leqslant\left(C_{0}-\delta\right) \phi_{y}$ in $\mathbb{R}_{+}^{2}$ and would contradict the minimality of $C_{0}$.

Let us first deal with $\Omega_{1}^{\prime}$. Since $f^{\prime}(\phi)=0$ in $\mathcal{C}_{-}(0, \alpha)$, both even (in $x$ ) functions $U(t)(x, y)$ and $\left(C_{0}-\delta\right) \phi_{y}(x, y)$ satisfy

$$
v_{t}-\Delta v+c \partial_{y} v=0 \quad \text { for all }(t, x, y) \in \mathbb{R} \times \mathcal{C}_{-}(0, \alpha),
$$

and $\operatorname{Re}(u) \leqslant\left(C_{0}-\delta\right) \phi_{y}$ on $\partial \mathcal{C}_{-}(0, \alpha)$. Let $\varepsilon^{*}$ be the smallest nonnegative $\varepsilon$ such that $\operatorname{Re}(u) \leqslant\left(C_{0}-\delta\right) \phi_{y}+\varepsilon$ in $\mathcal{C}_{-}(0, \alpha)$. Assume $\varepsilon^{*}>0$. Since $|u| \leqslant C \phi_{y}$, one knows that $\lim _{y \rightarrow-\infty} \sup _{\mathcal{C}_{-}(y, \alpha)}\left|\operatorname{Re}(u)-\left(C_{0}-\delta\right) \phi_{y}\right|=0$. Therefore, we may assume the existence of some sequence $\varepsilon_{n} \rightarrow \varepsilon^{*}$ and $\left(X_{n}, Y_{n}\right) \in \mathcal{C}_{-}(0, \alpha)$ such that $X_{n} \rightarrow+\infty$ and $Y_{n} \rightarrow Y_{\infty}<0$ such that

$$
\operatorname{Re}(u)\left(X_{n}, Y_{n}\right)-\left(C_{0}-\delta\right) \phi_{y}\left(X_{n}, Y_{n}\right)-\varepsilon_{n} \rightarrow 0 \quad \text { as } n \rightarrow+\infty .
$$

Arguing as in case 1 above and in the proof of Proposition 3.1, one then gets a contradiction with the positivity of $\varepsilon^{*}$.

Therefore, $\varepsilon^{*}=0$ and $\operatorname{Re}(u) \leqslant\left(C_{0}-\delta\right) \phi_{y}$ in $\mathcal{C}_{-}(0, \alpha)$.

Similarly, using the fact that

$$
f^{\prime}(\phi) \leqslant 0
$$

in $\left\{(x, y),(x, y) \in \Omega_{2}^{\prime}\right.$ or $\left.(-x, y) \in \Omega_{2}^{\prime}\right\}=\mathcal{C}_{+}\left(Y_{1} / \sin \alpha, \pi-\alpha\right)$, one can prove that

$$
\operatorname{Re}(u) \leqslant\left(C_{0}-\delta\right) \phi_{y} \quad \text { in } \mathcal{C}_{+}\left(Y_{1} / \sin \alpha, \pi-\alpha\right) .
$$

Eventually, $\operatorname{Re}(u) \leqslant\left(C_{0}-\delta\right) \phi_{y}$ in $\mathbb{R}^{2}$ for all $\delta>0$ small enough. This contradicts the definition of $C_{0}$.

Therefore, $C_{0} \leqslant 0$ and $\operatorname{Re}(u) \leqslant 0$. We may prove that $\operatorname{Re}(u) \geqslant 0$ in the same fashion, which implies $\operatorname{Re}(u)=0$, and then $u=0$ since $L u=i \omega u$.

Proof of Theorem 4.1. - It remains to prove that $L$ is a Fredholm operator; namely

$$
L=T+K, \quad \operatorname{Re}(\sigma(T)) \geqslant \beta \quad \text { for some } \beta>0, \quad K T^{-1} \text { compact. }
$$

To do so, we wish to find a weight function $p(x, y)$ such that the operator $M$, defined by

$$
L=p M(p I)
$$

is a second order elliptic operator whose zero-order coefficient is positive and bounded away from 0 outside a compact subset. A natural choice would be - at least in the right half-space

$$
p(x, y)=e^{-\rho X} \phi_{0}^{\prime}(Y)
$$

where $\rho>0$ is small and $X, Y$ are the rotated coordinates. Such a choice would almost work, up to the fact that we are here asking too much decay at infinity. Therefore the weight will have to be slightly modified, in order to keep only the decay that is asked to functions belonging to $G_{\rho}$.

In the sequel of the proof of Theorem 4.1, we fix $\rho \in\left(0, c_{0} \cot \alpha\right)$, and call

$$
\lambda=\frac{\rho\left(c_{0} \cot \alpha-\rho\right)}{4}>0 .
$$


Step 1 (an auxiliary function). Let $\phi_{0}$ be the 1D wave, and let the real number $t_{+}$be chosen so that $\phi\left(x+x_{n}, y-\left|x_{n}\right| \cot \alpha\right) \rightarrow \phi_{0}\left( \pm x \cos \alpha+y \sin \alpha+t_{+}\right)$as $x_{n} \rightarrow \pm \infty$ (we recall that $\phi$ is assumed to be even in the variable $x$ ). Set

$$
L_{0}=-\partial_{Y}^{2}+c_{0} \partial_{Y}-f^{\prime}\left(\phi_{0}\left(.+t_{+}\right)\right)
$$

It is known that $\phi_{0}^{\prime}>0$ in $\mathbb{R}, \phi_{0}^{\prime \prime}(s) / \phi_{0}^{\prime}(s) \rightarrow c_{0}$ (respectively $\rightarrow-\mu$ ) as $s \rightarrow-\infty$ (respectively as $s \rightarrow+\infty$ ), and $\phi_{0}^{\prime \prime \prime}(s) / \phi_{0}^{\prime}(s) \rightarrow c_{0}^{2}$ (respectively $\rightarrow \mu^{2}$ ) as $s \rightarrow-\infty$ (respectively as $s \rightarrow+\infty)$, where $\mu=\left(c_{0}+\sqrt{c_{0}^{2}-4 f^{\prime}\left(1^{-}\right)}\right) / 2$.

Lastly, one has that $f^{\prime}\left(\phi_{0}(s)\right)=0$ for $-s$ large enough, and $f^{\prime}\left(\phi_{0}(s)\right) \rightarrow f^{\prime}\left(1^{-}\right)$as $s \rightarrow+\infty$. Therefore, there exist $A>0$ and a function $\psi$ of class $C^{2}$ such that

$$
\begin{cases}\forall|Y| \leqslant A, & \psi(Y)=\phi_{0}^{\prime}\left(Y+t_{+}\right), \\ \forall|Y| \geqslant 2 A, & \psi^{\prime}(Y)=0, \\ \forall-2 A \leqslant Y \leqslant-A, & -\frac{\psi^{\prime \prime}(Y)}{\psi(Y)}+c_{0} \frac{\psi^{\prime}(Y)}{\psi(Y)}-f^{\prime}\left(\phi_{0}\left(Y+t_{+}\right)\right) \geqslant-\lambda, \\ \forall A \leqslant Y \leqslant 2 A, & -\frac{\psi^{\prime \prime}(Y)}{\psi(Y)}+c_{0} \frac{\psi^{\prime}(Y)}{\psi(Y)}-f^{\prime}\left(\phi_{0}\left(Y+t_{+}\right)\right) \geqslant-\lambda, \\ \min _{\mathbb{R}} \psi>0 . & \end{cases}
$$

The existence of such a function $\psi$ can be obtained through a slight perturbation of the exponential tails of $\phi_{0}^{\prime}$. We call $C_{0}$ a positive constant such that

$$
\left\|\frac{\psi^{\prime}}{\psi}\right\|_{L^{\infty}(\mathbb{R})}+\left\|\frac{\psi^{\prime \prime}}{\psi}\right\|_{L^{\infty}(\mathbb{R})} \leqslant C_{0} .
$$

We also choose two $C^{\infty}$ functions $k_{1}$ and $k_{2}: \mathbb{R} \rightarrow \mathbb{R}$ such that $0 \leqslant k_{1}, k_{2} \leqslant 1, k_{1}=k_{2}=1$ on $[-A, A]$ and $k_{1}=k_{2}=0$ outside $[-2 A, 2 A]$.

Step 2 (construction of $T$ ). We next choose a $C^{\infty}$ convex function $h: \mathbb{R} \rightarrow \mathbb{R}$ such that $h(x)=|x|$ for $|x|$ large enough, and

$$
0 \leqslant h^{\prime \prime} \leqslant \lambda /\left(C_{0} \cos \alpha\right)
$$

where $C_{0}$ is as above. Notice that the above properties especially imply that $\left|h^{\prime}\right| \leqslant 1$.

We set, only in this particular step 2:

$$
\begin{aligned}
& X=h(x) \sin \alpha-y \cos \alpha, \\
& Y=h(x) \cos \alpha+y \sin \alpha .
\end{aligned}
$$

Since $f^{\prime}\left(\phi_{0}\left(Y+t_{+}\right)\right)-f^{\prime}(\phi(x, y)) \rightarrow 0$ uniformly as $|(x, y)| \rightarrow+\infty$, and

$$
\lim _{y_{0} \rightarrow+\infty} \sup _{y \geqslant y_{0}-|x| \cot \alpha}\left|f^{\prime}(\phi(x, y))-f^{\prime}\left(1^{-}\right)\right|=\lim _{y_{0} \rightarrow-\infty} \sup _{y \leqslant y_{0}-|x| \cot \alpha}\left|f^{\prime}(\phi(x, y))\right|=0,
$$

one may, without loss of generality, choose $A$ large enough so that

$$
\left[f^{\prime}\left(\phi_{0}\left(Y+t_{+}\right)\right)\left(1-k_{1}(x)\right)-f^{\prime}(\phi(x, y))\right]\left(1-k_{1}(x) k_{2}(Y)\right) \geqslant-\lambda
$$

for all $(x, y) \in \mathbb{R}^{2}$. 
We finally set

$$
u(x, y)=p(x, y) v(x, y)
$$

where

$$
p(x, y)=e^{-\rho X} \psi(Y) .
$$

Let us write, for every $C^{2}$ function $u$ :

$$
L u(x, y)=p(x, y) M v(x, y)
$$

The operator $M$ has the form

$$
M=-\Delta+B(x, y) \cdot \nabla+\frac{L p}{p}
$$

where $B=-2 \nabla p / p+(0, c)$ is a $C^{1}$ bounded vector-valued function.

Let us now evaluate $L p$. Using (1.5) and the fact that $c=c_{0} / \sin \alpha$, a straightforward calculation gives:

$$
\frac{L p}{p}=a(x, y)+b(x, y)
$$

where

$$
\left\{\begin{aligned}
a(x, y)= & c_{0} \rho \cot \alpha-\rho^{2} \sin ^{2} \alpha{h^{\prime}}^{2}(x) \\
& +\left[-\frac{\psi^{\prime \prime}(Y)}{\psi(Y)}+c_{0} \frac{\psi^{\prime \prime}(Y)}{\psi(Y)}-f^{\prime}\left(\phi_{0}\left(Y+t_{+}\right)\right)\right]\left(1-k_{1}(x)\right) \\
& +\left[f^{\prime}\left(\phi_{0}\left(Y+t_{+}\right)\right)\left(1-k_{1}(x)\right)-f^{\prime}(\phi(x, y))\right]\left(1-k_{1}(x) k_{2}(Y)\right) \\
& +\left(\rho \sin \alpha-\cos \alpha \frac{\psi^{\prime}(Y)}{\psi(Y)}\right) h^{\prime \prime}(x) \\
b(x, y)= & \left.-\frac{\psi^{\prime \prime}(Y)}{\psi(Y)}+c_{0} \frac{\psi^{\prime \prime}(Y)}{\psi(Y)}\right] k_{1}(x) \\
& +\cos \alpha\left[\cos \alpha \frac{\psi^{\prime \prime}(Y)}{\psi(Y)}-2 \rho \sin \alpha \frac{\psi^{\prime}(Y)}{\psi(Y)}\right]\left(1-h^{\prime 2}(x)\right) \\
& +\left[f^{\prime}\left(\phi_{0}\left(Y+t_{+}\right)\right)\left(1-k_{1}(x)\right)-f^{\prime}(\phi(x, y))\right] k_{1}(x) k_{2}(Y) .
\end{aligned}\right.
$$

The function $a$ is clearly continuous and bounded in $\mathbb{R}^{2}$. Let us now estimate it from below. Assume that $A>0$ is large enough so that

$$
f^{\prime}\left(\phi\left(s+t_{+}\right)\right)=0 \quad \text { for } s \leqslant-2 A, \quad-f^{\prime}\left(\phi\left(s+t_{+}\right)\right) \geqslant-f^{\prime}\left(1^{-}\right) / 2 \geqslant 0 \quad \text { for } s \geqslant 2 A .
$$

It follows then from the choice of $\psi$ that

$$
\left[-\frac{\psi^{\prime \prime}(Y)}{\psi(Y)}+c_{0} \frac{\psi^{\prime \prime}(Y)}{\psi(Y)}-f^{\prime}\left(\phi_{0}\left(Y+t_{+}\right)\right)\right]\left(1-k_{1}(x)\right) \geqslant-\lambda\left(1-k_{1}(x)\right) \geqslant-\lambda
$$

for all $(x, y) \in \mathbb{R}^{2}$. Putting the above estimate together with (4.10), (4.9) and the fact that $\left|h^{\prime}\right| \leqslant 1$, it follows that

$$
\forall(x, y) \in \mathbb{R}^{2}, \quad a(x, y) \geqslant \rho\left(c_{0} \cot \alpha-\rho\right)-3 \lambda=\lambda>0
$$


On the other hand, it follows from the choices of $h, k_{1}$ and $k_{2}$ that the function $b$ is continuous with compact support in $\mathbb{R}^{2}$.

Set

$$
\tilde{M}=-\Delta+B(x, y) \cdot \nabla+a(x, y)
$$

and

$$
T u=p \tilde{M}\left(\frac{u}{p}\right) ; \quad K u=L u-T u=b(x, y) u .
$$

Step 3. Let us prove the existence of $\beta>0$ such that $\operatorname{Re}(\sigma(T)) \geqslant \beta$. For this we estimate $\left\|e^{-t T}\right\|_{\mathcal{L}\left(G_{\rho}\right)}$. Let $u_{0} \in G_{\rho}$. We have

$$
e^{-t T} u_{0}=p e^{-t \tilde{M}}\left(\frac{u_{0}}{p}\right)
$$

and the maximum principle yields

$$
\left\|p^{-1} e^{-t T} u_{0}\right\|_{\infty} \leqslant e^{-\lambda t}\left\|p^{-1} u_{0}\right\|_{\infty} \leqslant C e^{-\lambda t}\left\|q^{-1} u_{0}\right\|_{\infty},
$$

where $q$ was defined in (1.9) and $C$ is a constant which does not depend on $u_{0}$. We here use the definition of $G_{\rho}$, and the fact that the function $\psi$ is bounded from below by a positive constant. Furthermore, since $\psi$ is bounded from above, one infers that

$$
\left\|q^{-1} e^{-t T} u_{0}\right\|_{\infty} \leqslant C^{\prime}\left\|p^{-1} e^{-t T} u_{0}\right\|_{\infty}
$$

for some constant $C^{\prime}>0$. Hence,

$$
\left\|q^{-1} e^{-t T} u_{0}\right\|_{\infty} \leqslant C C^{\prime} e^{-\lambda t}\left\|q^{-1} u_{0}\right\|_{\infty}
$$

On the other hand, let us choose $B>0$ large enough so that $b=0$ and $f^{\prime}(\phi) \leqslant f^{\prime}\left(1^{-}\right) / 2<0$ in $\mathcal{C}_{+}(B, \pi-\alpha)$. Observe now that the function $q$ is bounded in $\mathcal{C}_{-}(B, \alpha)$. Therefore, there exists a constant $C^{\prime \prime}$ (independent of $u_{0}$ ) such that

$$
\left\|e^{-t T} u_{0}\right\|_{L^{\infty}\left(\mathcal{C}_{-}(B, \alpha)\right)} \leqslant C^{\prime \prime}\left\|q^{-1} e^{-t T} u_{0}\right\|_{L^{\infty}\left(\mathcal{C}_{-}(B, \alpha)\right)} \leqslant C C^{\prime} C^{\prime \prime} e^{-\lambda t}\left\|q^{-1} u_{0}\right\|_{\infty}
$$

Lastly, the function $u(t):=e^{-t T} u_{0}$ satisfies

$$
\begin{cases}u_{t}-\Delta u+c u_{y}-f^{\prime}(\phi) u=0 & \text { in } \mathcal{C}_{+}(B, \pi-\alpha), \\ |u(t, x, y)| \leqslant C C^{\prime} C^{\prime \prime} e^{-\lambda t}\left\|q^{-1} u_{0}\right\|_{\infty} & \text { on } \partial \mathcal{C}_{+}(B, \pi-\alpha) .\end{cases}
$$

Hence we have

$$
\left\|e^{-t T} u_{0}\right\|_{L^{\infty}\left(\mathcal{C}_{+}(B, \pi-\alpha)\right)} \leqslant\left(1+C C^{\prime} C^{\prime \prime}\right) e^{-\beta t}\left(\left\|u_{0}\right\|_{L^{\infty}\left(\mathcal{C}_{+}(B, \pi-\alpha)\right)}+\left\|q^{-1} u_{0}\right\|_{\infty}\right),
$$

where $\beta=\min \left(\lambda,-f^{\prime}\left(1^{-}\right) / 2\right)>0$.

Summing up, one gets that

$$
\left\|e^{-t T} u_{0}\right\|_{G_{\rho}} \leqslant \tilde{C} e^{-\beta t}\left\|u_{0}\right\|_{G_{\rho}}
$$


for some constant $\tilde{C}$. Therefore - by a standard Laplace transform argument - the spectrum of $L$ satisfies $\operatorname{Re}(\sigma(T)) \geqslant \beta$.

Step 4 (conclusion). For every $\lambda$ such that $\operatorname{Re}(\lambda) \in(-\infty, \beta)$, the operator $T-\lambda I$ is an isomorphism of a dense subspace of $G_{\rho}$ onto $G_{\rho}$, and $K(T-\lambda I)^{-1}$ is compact. Moreover, $L$ is sectorial in $G_{\rho}$ (Stewart [39]).

Combining these considerations with Propositions 4.2-4.5, we obtain the existence of a cone with aperture less than $\pi / 2$ and positive vertex containing the spectrum of $L-$ see [29] for more details. Classical stability results [22] apply subsequently.

\section{Convergence to a single wave}

The aim of this section is to prove Theorems 1.6 and 1.7. In this section we keep the notations of the preceding section. In particular, we use the rotated coordinate system $(X, Y)$. We will have to investigate the behaviour of different functions as the space variable becomes infinite along the directions $e_{\alpha}=(\sin \alpha,-\cos \alpha)$ and $e_{\alpha}^{\prime}=(-\sin \alpha,-\cos \alpha)$. Only the direction $e_{\alpha}$ will be investigated, the case of $e_{\alpha}^{\prime}$ being similar.

The first result that we need is another Liouville type property.

Proposition 5.1. - Let $v(t, X, Y)$ ranging in $[0,1]$ be a classical solution of

$$
\begin{aligned}
v_{t}-\Delta v-c_{0} \cot \alpha v_{X}+c_{0} v_{Y} & =f(v), \quad(t, X, Y) \in \mathbb{R}^{3}, \\
& \lim \sup _{Y \rightarrow-\infty,(t, X) \in \mathbb{R}^{2}} v(t, X, Y)=0, \\
& \liminf _{Y \rightarrow+\infty,(t, X) \in \mathbb{R}^{2}} v(t, X, Y)>\theta .
\end{aligned}
$$

Then there exists $Y_{0} \in \mathbb{R}$ such that $v(t, X, Y)=\phi_{0}\left(Y+Y_{0}\right)$ for all $(t, X, Y) \in \mathbb{R}^{3}$.

Proof. - The first part of the proof consists in observing that there exists $Y_{1} \in \mathbb{R}$ and $\eta \in(\theta, 1]$ such that $v(t, X, Y) \geqslant H\left(Y+Y_{1}\right)$ for all $(t, X, Y) \in \mathbb{R}^{3}$, where $H(s)=0$ if $s<0$ and $H(s)=\eta$ if $s \geqslant 0$. Therefore, arguing as in the proof of Theorem 1.5, one gets the existence of $Y_{2} \in \mathbb{R}$ such that $v(t, X, Y) \geqslant \phi_{0}\left(Y+Y_{2}\right)$ for all $(t, X, Y) \in \mathbb{R}^{3}$.

The second part of the proof is identical to the proof of Theorem 1.3.

Let $u_{0}$ and $\phi$ satisfy the assumptions of Theorem 1.6, and let $u(t, x, y)$ be the solution of (1.3). Up to a same shift of both $u_{0}$ and $\phi$, we may assume without loss of generality that $\phi(0,0)=\theta$ and $\phi$ is even in $x$. A standard argument from local existence theory for nonlinear parabolic equations - see [22], Chapter 3, and [1] - would yield the exponential spatial convergence of the solution $u$ under investigation to a 1D wave in the $X$ direction, locally in $Y$. Notice especially that, for the function $\phi$, there exists $t_{+} \in \mathbb{R}$ such that, for all $K>0$, there are $C_{K}>0$ and $\lambda_{K}>0$ such that

$$
\forall X \geqslant 0, \forall|Y| \leqslant K, \quad\left|\phi(X, Y)-\phi_{0}\left(Y+t_{+}\right)\right| \leqslant C_{K} e^{-\lambda_{K} X} .
$$

The same type of property holds in the left plane $\{x<0\}$.

As far as the function $u(t, x, y)$ is concerned, such an exponential decay is a priori not uniform in time, and our point is that this convergence is indeed uniform in time. This is the goal of the next proposition.

PROPOSITION 5.2. - Under the assumptions of Theorem 1.6, there are constants $C>0$, $t_{0}>0$ and $\rho_{1}>0$, such that

$$
\left|\partial_{e_{\alpha}} u(t, x, y-c t)\right| \leqslant C e^{-\rho_{1} X+c_{0} Y / 2}, \quad\left|\partial_{e_{\alpha}} u(t,-x, y-c t)\right| \leqslant C e^{-\rho_{1} X+c_{0} Y / 2}
$$

for all $t \geqslant t_{0}$ and $(x, y) \in \mathbb{R}_{+}^{2}$, where $X=x \sin \alpha-y \cos \alpha, Y=x \cos \alpha+y \sin \alpha$. 
Proof. - It is divided into several steps.

Step 1 (estimates for $u$ ). Set

$$
\bar{u}(t, x, y)=u(t, x, y-c t) .
$$

The function $\bar{u}$ satisfies $\bar{u}_{t}=\Delta \bar{u}-c \bar{u}_{y}+f(\bar{u})$ and $0 \leqslant \bar{u}(t, x, y) \leqslant \tau_{a, b} \phi(x, y)$ for all $t \geqslant 0$ and $(x, y) \in \mathbb{R}^{2}$. Since both functions $\phi$ and

$$
w(x, y)=e^{c_{0}(x \cos \alpha+y \sin \alpha)}+e^{c_{0}(-x \cos \alpha+y \sin \alpha)}
$$

satisfy $\Delta v-c v_{y}=0$ in $\mathcal{C}_{-}(0, \alpha)$, together with $\lim _{y \rightarrow-\infty} \sup _{\mathcal{C}_{-}(y, \alpha)} \phi($ respectively $w)=0$ and $\phi \leqslant \theta \leqslant w$ on $\partial \mathcal{C}_{-}(0, \alpha)$, it follows from Lemma 5.1 in [19] that

$$
\phi \leqslant w \quad \text { in } \mathcal{C}_{-}(0, \alpha)
$$

On the other hand, $\nabla_{x, y} \bar{u}$, as well as $\partial_{t} \nabla_{x, y} \bar{u}$ and the spatial derivatives of $\nabla_{x, y} \bar{u}$ up to the second order, are globally bounded for all $t \geqslant 1$ and $(x, y) \in \mathbb{R}^{2}$. Furthermore, the function $w$ is bounded from below by a positive constant in any strip of the type $\mathcal{C}_{+}(B, \pi-\alpha) \backslash \mathcal{C}_{-}(A, \alpha)$ for each $A<B$. Standard parabolic estimates then imply that there exist some constants $C_{1}$ and $C_{1}^{\prime}\left(y_{0}\right)$ such that

$$
\begin{aligned}
& \left|\nabla_{x, y} \bar{u}(t, x, y)\right|+\left|\partial_{t} \nabla_{x, y} \bar{u}(t, x, y)\right|+\left|D^{2} \bar{u}(t, x, y)\right|+\left|D^{3} \bar{u}(t, x, y)\right| \\
& \quad \leqslant \begin{cases}C_{1}^{\prime}\left(y_{0}\right)\left(e^{c_{0}(x \cos \alpha+y \sin \alpha)}+e^{c_{0}(-x \cos \alpha+y \sin \alpha)}\right) & \text { in } \mathcal{C}_{-}\left(y_{0}, \alpha\right), \\
C_{1} & \text { in } \mathbb{R}^{2}\end{cases}
\end{aligned}
$$

and for all $t \geqslant 1$, where $\left|D^{2} \bar{u}\right|$ and $\left|D^{3} \bar{u}\right|$ respectively mean the maximum of the absolute values of the second order (respectively third order) spatial derivatives of $\bar{u}$.

Step 2 (estimates for $\phi_{X}$ ). First of all, it follows from (5.2) and standard elliptic estimates that

$$
\left|\phi_{X}\right| \leqslant C_{2} e^{-\lambda X} \quad \text { on }\{y=-x \cot \alpha, x \geqslant 0\}=\{X \geqslant 0, Y=0\}
$$

for some $\lambda>0$. Similar estimates as (5.3) obviously hold for the derivatives of $\phi$ in $\mathcal{C}_{-}(0, \alpha)$. Therefore, even if it means increasing $C_{2}>0$, decreasing $\lambda>0$, one can assume that

$$
\left|\phi_{X}\right| \leqslant C_{2} e^{-\lambda X+\frac{3 c_{0}}{4}(x \cos \alpha+y \sin \alpha)}=: v(x, y) \quad \text { on } \partial\left(\mathcal{C}_{-}(0, \alpha) \cap \mathbb{R}_{+}^{2}\right) .
$$

A direct calculation shows that $v$ satisfies $\Delta v-c v_{y} \leqslant 0$ in $\mathcal{C}_{-}(0, \alpha) \cap \mathbb{R}_{+}^{2}$, as soon as $\lambda^{2}-c_{0} \lambda \cot \alpha \leqslant 3 c_{0}^{2} / 16$, which can always be assumed even if it means decreasing $\lambda$. Since

$$
\limsup _{\operatorname{dist}\left((x, y), \partial\left(\mathcal{C}_{-}(0, \alpha) \cap \mathbb{R}_{+}^{2}\right)\right) \rightarrow+\infty,(x, y) \in \mathcal{C}_{-}(0, \alpha) \cap \mathbb{R}_{+}^{2}}\left|\phi_{X}(x, y)\right|=0
$$

and $v \geqslant 0$, it follows therefore from the proof of Lemma 5.1 in $[19]^{4}$ that $\left|\phi_{X}\right| \leqslant v$ in $\mathcal{C}_{-}(0, \alpha) \cap \mathbb{R}_{+}^{2}$. In other words,

$$
\left|\phi_{X}(x, y)\right| \leqslant C_{2} e^{-\lambda X+\frac{3 c_{0}}{4} Y} \quad \text { in } \mathcal{C}_{-}(0, \alpha) \cap \mathbb{R}_{+}^{2} .
$$

\footnotetext{
${ }^{4}$ The proof can easily be adapted to our situation, the boundary of $\mathcal{C}_{-}(0, \alpha) \cap \mathbb{R}_{+}^{2}$ being a Lipschitz graph in a rotated frame.
} 
On the other hand, because of (1.2) and since $f^{\prime}\left(1^{-}\right)<0$, there exists $y_{1}$ such that $f^{\prime}(\phi) \leqslant f^{\prime}\left(1^{-}\right) / 2<0$ in $\mathcal{C}_{+}\left(y_{1}, \pi-\alpha\right)$. Even if it means decreasing $\lambda>0$, the function $\zeta:=e^{-\lambda X+\lambda Y}$ satisfies

$$
\Delta \zeta-c \partial_{y} \zeta+f^{\prime}(\phi) \zeta \leqslant 0 \quad \text { in } \mathcal{C}_{+}\left(y_{1}, \pi-\alpha\right)
$$

while $\left|\phi_{X}\right| \leqslant C_{3} \zeta$ on $\partial\left(\mathcal{C}_{+}\left(y_{1}, \pi-\alpha\right) \cap \mathbb{R}_{+}^{2}\right)$ for some constant $C_{3}$. Since

$$
\lim _{y \rightarrow+\infty} \sup _{\mathcal{C}_{+}(y, \pi-\alpha)}\left|\phi_{X}\right|=0
$$

it follows from the proof of Lemma 5.1 in [19] that

$$
\left|\phi_{X}\right| \leqslant C_{3} \zeta=C_{3} e^{-\lambda X+\lambda Y} \quad \text { in } \mathcal{C}_{+}\left(y_{1}, \pi-\alpha\right) \cap \mathbb{R}_{+}^{2} .
$$

Step 3 (estimates for $\left.\bar{u}_{X}-\phi_{X}\right)$. The function $z(t, x, y):=\bar{u}(t, x, y)-\phi(x, y)$ satisfies an equation of the type

$$
\partial_{t} z-\Delta z+c \partial_{y} z+\gamma(t, x, y) z=0,
$$

where $\gamma$ is bounded and $\|\gamma\|_{L^{\infty}\left((0,+\infty) \times \mathbb{R}^{2}\right)} \leqslant\|f\|_{\text {Lip }}\left(\|f\|_{\text {Lip }}\right.$ denotes the Lipschitz norm of $f$ ). Choose now any direction $\nu$ of $\mathbb{R}^{2}$ such that $|\nu|=1$. It follows from the assumptions of Theorem 1.6 that $|z(0, x, y)| \leqslant C_{0} e^{-\rho_{0} \nu \cdot(x, y)}$ in $\mathbb{R}^{2}$. Let $\omega_{0}=\rho_{0}^{2}+c \rho_{0}+\|f\|_{\text {Lip. }}$. It is easy to check that the function $\kappa(t, x, y):=C_{0} e^{\omega_{0} t-\rho_{0} \nu \cdot(x, y)}$ satisfies

$$
\partial_{t} \kappa-\Delta \kappa+c \partial_{y} \kappa-\|f\|_{\text {Lip }} \kappa \geqslant 0
$$

together with $\kappa(0, x, y) \geqslant|z(0, x, y)|$ in $\mathbb{R}^{2}$. The maximum principle then yields that $|z(t, x, y)| \leqslant \kappa(t, x, y)$, whence

$$
|\bar{u}(t, x, y)-\phi(x, y)| \leqslant C_{0} e^{\omega_{0} t-\rho_{0} \nu \cdot(x, y)}
$$

for all $t \geqslant 0$ and $(x, y) \in \mathbb{R}^{2}$. Since the above estimate holds for all $\nu \in \mathbb{R}^{2}$ with $|\nu|=1$, one concludes that

$$
|\bar{u}(t, x, y)-\phi(x, y)| \leqslant C_{0} e^{\omega_{0} t-\rho_{0} \sqrt{x^{2}+y^{2}}}
$$

for all $t \geqslant 0$ and $(x, y) \in \mathbb{R}^{2}$.

Standard parabolic estimates then imply that

$$
\left|\bar{u}_{X}(t, x, y)-\phi_{X}(x, y)\right| \leqslant C_{4} e^{\omega_{0} t-\rho_{0} \sqrt{x^{2}+y^{2}}}
$$

for all $t \geqslant 1$ and $(x, y) \in \mathbb{R}^{2}$, for some constant $C_{4}$.

Furthermore, estimates of the type (5.3) also hold by replacing $\bar{u}$ with $\phi$ (take $u_{0}=\phi$ as the initial condition). Therefore,

$$
\left|\bar{u}_{X}(t, x, y)-\phi_{X}(x, y)\right| \leqslant C_{4}^{\prime}\left(e^{c_{0}(x \cos \alpha+y \sin \alpha)}+e^{c_{0}(-x \cos \alpha+y \sin \alpha)}\right)
$$

in $\mathcal{C}_{-}(0, \alpha)$, for all $t \geqslant 1$, and for some constant $C_{4}^{\prime}$.

Step 4 (auxiliary functions and definition of a set $\Omega^{\prime}$ ). Choose now some positive coefficients $\rho_{1}, \rho_{2}$ and $c_{1}$ such that $0<\rho_{2}<\rho_{1}, 0<c_{1}<c_{0}$ and $2\left(\rho_{1}+\rho_{2}\right)<\left(c_{0}-c_{1}\right) \tan \alpha$. Consider the 
function

$$
v_{1}(t, x, y)=e^{\rho_{1} X-c_{0} Y / 2} \bar{u}_{X}(t, x, y)
$$

defined for all $t>0$ and $(x, y) \in \mathbb{R}^{2}$, and let $\Omega^{\prime}$ be the set defined by

$$
\Omega^{\prime}=\left\{(x, y) \in \mathbb{R}^{2}, x>-1 \text { and }\left(X>X_{1} \text { or } Y>Y_{1}\right)\right\}
$$

where $X_{1}>0$ and $Y_{1}>0$ shall be chosen below.

From the above upper bounds for $\left|\nabla_{x, y} \bar{u}\right|$ given in step 1, it is straightforward to check that there is a constant $C_{5}=C_{5}\left(X_{1}, Y_{1}\right)>0$ such that

$$
\begin{gathered}
\left|v_{1}(t, x, y)\right|+\left|\partial_{t} v_{1}(t, x, y)\right|+\left|\nabla_{x, y} v_{1}(t, x, y)\right| \\
\quad+\left|D^{2} v_{1}(t, x, y)\right| \leqslant C_{5} e^{-\rho_{2}|X|-c_{1}|Y| / 2}
\end{gathered}
$$

for all $t \geqslant 1$ and $(x, y) \in \partial \Omega^{\prime}$ (remember that the quantity $x$ is bounded on $\partial \Omega^{\prime}$ ). Note that (5.9) is not optimal when $Y$ (or $y$ ) becomes positive and large; all we need, however, is an integrability condition for $v_{1}$.

Set $\psi(t, x, y)=v_{1}(t, x, y)$ for all $t \geqslant 1$ and $(x, y) \in \partial \Omega^{\prime}$ and extend $\psi$ in $[1,+\infty) \times \bar{\Omega}^{\prime}$ by a $C^{2}$ function, still denoted by $\psi$, such that $\psi$, as well as $\psi_{t}$ and the space derivatives of $\psi$ up to the second order, are bounded by $C_{6} e^{-\rho_{2}|X|-c_{1}|Y| / 2}$ in $[1,+\infty) \times \bar{\Omega}^{\prime}$ for some constant $C_{6}$. Finally set, for all $t \geqslant 1$ and $(x, y) \in \bar{\Omega}^{\prime}$,

$$
v(t, x, y)=v_{1}(t, x, y)-\psi(t, x, y)
$$

Step $5\left(v(t, \cdot, \cdot) \in L^{2}\left(\Omega^{\prime}\right)\right.$ for each $\left.t \geqslant 1\right)$. First, the function $\psi(t, \cdot, \cdot)$ is in $L^{2}\left(\Omega^{\prime}\right)$ by construction. Write now

$$
v_{1}(t, x, y)=v_{2}(x, y)+v_{3}(t, x, y),
$$

where $v_{2}(x, y)=e^{\rho_{1} X-c_{0} Y / 2} \phi_{X}(x, y)$ and $v_{3}(t, x, y)=e^{\rho_{1} X-c_{0} Y / 2}\left(\bar{u}_{X}(t, x, y)-\phi_{X}(x, y)\right)$.

The function $v_{2}$ is in $L^{2}\left(\Omega^{\prime} \cap \mathcal{C}_{-}(0, \alpha)\right)$ because of (5.4), even if it means decreasing $\rho_{1}$ so that

$$
0<\rho_{1}<\lambda .
$$

One has $v_{2} \in L^{2}\left(\Omega^{\prime} \cap\left\{0 \leqslant Y \leqslant y_{1} / \sin \alpha\right\}\right)$ because of (5.2) and (5.10). On the other hand, $v_{2} \in L^{2}\left(\Omega^{\prime} \cap\left\{Y \geqslant y_{1} / \sin \alpha, X \geqslant 0\right\}\right)$ because of (5.5), (5.10), and even if it means decreasing $\lambda$ so that $0<\lambda<c_{0} / 2$. Lastly, $v_{2} \in L^{2}\left(\Omega^{\prime} \cap\{X \leqslant 0\}\right)$ because $\phi_{X}$ is globally bounded. Therefore, $v_{2} \in L^{2}\left(\Omega^{\prime}\right)$.

Fix now a real number $\beta>0$ such that $\rho_{0}>c_{0} \beta / 2$ and $\beta<\tan \alpha$. Let $t \geqslant 1$. The function $v_{3}(t, \cdot, \cdot)$ is in $L^{2}\left(\Omega^{\prime} \cap\{Y \leqslant-\beta X\}\right)$ because of (5.8), even if it means decreasing $\rho_{1}$ so that $0<\rho_{1}<c_{0} \beta / 2$. The function $v_{3}(t, \cdot, \cdot)$ is in $L^{2}\left(\Omega^{\prime} \cap\{|Y| \leqslant \beta X\}\right)$ because of (5.7), even if it means decreasing $\rho_{1}$ so that $0<\rho_{1}<\rho_{0}-c_{0} \beta / 2$. On the other hand, $v_{3}(t, \cdot, \cdot) \in L^{2}\left(\Omega^{\prime} \cap\right.$ $\{Y \geqslant \beta X, X \geqslant 0\})$ because $u_{X}-\phi_{X}$ is globally bounded in $L^{\infty}\left([1,+\infty) \times \mathbb{R}^{2}\right)$ and because $0<\rho_{1}<c_{0} \beta / 2$. Lastly, $v_{3}(t, \cdot, \cdot) \in L^{2}\left(\Omega^{\prime} \cap\{X \leqslant 0\}\right)$ because $u_{X}-\phi_{X}$ is globally bounded in $L^{\infty}\left([1,+\infty) \times \mathbb{R}^{2}\right)$. Therefore, $v_{3}(t, \cdot, \cdot) \in L^{2}\left(\Omega^{\prime}\right)$ for each $t \geqslant 1$.

One concludes that $v(t, \cdot, \cdot) \in L^{2}\left(\Omega^{\prime}\right)$ for each $t \geqslant 1$.

Step 6 (integration by parts over $\Omega^{\prime}$ ). Multiply the equation for $v$ by $v$; integrate by parts over $\Omega^{\prime}$. We get 


$$
\frac{1}{2} \frac{d}{d t} \int_{\Omega^{\prime}} v^{2}=\underbrace{-\int_{\Omega^{\prime}}\left(v_{X}^{2}+\left(c_{0} \cot \alpha \rho_{1}-\rho_{1}^{2}\right) v^{2}\right)}_{\text {I }}-\underbrace{-\int\left(v_{Y}^{2}-\left(f^{\prime}(\bar{u})+\frac{c_{0}^{2}}{4}\right) v^{2}\right)}_{\text {II }}-\underbrace{\int_{\Omega^{\prime}}(P \psi) v}_{\text {III }}
$$

where $P$ is a parabolic operator with bounded coefficients. Let us analyse these three terms.

The term I is the one that will control the estimate of $v$. We may obviously estimate it by

$$
\mathrm{I} \leqslant-\left(c_{0} \cot \alpha \rho_{1}-\rho_{1}^{2}\right) \int_{\Omega^{\prime}} v^{2},
$$

and, even if it means decreasing both $\rho_{1}$ and $\rho_{2}$, we may assume that

$$
0<\rho_{1}<c_{0} \cot \alpha .
$$

By assumption, $\left|u_{0}(x, y)-\phi(x, y)\right|=\mathrm{O}\left(e^{-\rho_{0} \sqrt{x^{2}+y^{2}}}\right)$ as $x^{2}+y^{2} \rightarrow+\infty$, whence

$$
\lim _{y \rightarrow+\infty} \inf _{\mathcal{C}_{-}(y, \pi-\alpha)} u_{0}=1>\theta .
$$

From the proof of Theorem 1.5, there exist two functions $V^{ \pm}(t, s)$ such that

$$
\bar{u}(t, x, y) \geqslant \max \left(V^{ \pm}(t, \pm x \cos \alpha+y \sin \alpha)\right)
$$

for all $t \geqslant 0$ and $(x, y) \in \mathbb{R}^{2}$, where $V^{ \pm}(t, s)-\phi_{0}\left(s+s_{1}\right) \rightarrow 0$ uniformly in $s \in \mathbb{R}$ as $t \rightarrow+\infty$. In particular, it also follows that, for any $\varepsilon>0$, one has $\bar{u}(t, x, y) \geqslant 1-\varepsilon$ as soon as $Y$ and $t$ are large enough, uniformly in $X \in \mathbb{R}$. On the other hand, $u_{0} \leqslant \tau_{a, b} \phi$ implies that $\bar{u}(t, x, y) \leqslant \tau_{a, b} \phi(x, y)$ for all $t \geqslant 0$ and $(x, y) \in \mathbb{R}^{2}$. From Proposition 5.1, there is then a bounded function $t \mapsto Y_{t}$, defined for $t$ large enough, such that

$$
\lim _{X \rightarrow+\infty} \lim _{t \rightarrow+\infty}\left|\bar{u}(t, X, Y)-\phi_{0}\left(Y+Y_{t}\right)\right|=0
$$

uniformly in $Y \in \mathbb{R}$ (under the restriction that $x>-1$ ) - argue by contradiction.

As a consequence, there exist $X_{1}>0$ and $Y_{1}>0$ in the definition of $\Omega^{\prime}$ so that

$$
\left|f^{\prime}(\bar{u}(t, x, y))-f^{\prime}\left(\phi_{0}\left(Y+Y_{t}\right)\right)\right| \leqslant \frac{1}{3}\left(c_{0} \cot \alpha \rho_{1}-\rho_{1}^{2}\right) \quad \text { in } \Omega^{\prime}
$$

for $t$ large enough (remember that $0<\rho_{1}<c_{0} \cot \alpha$ ).

On the other hand we have, for all $a \in \mathbb{R}$, for all $V \in H_{0}^{1}(a,+\infty)$, and as long as $Y_{t}$ is defined:

$$
\int_{a}^{+\infty}\left(V_{Y}^{2}-\left(f^{\prime}\left(\phi_{0}\left(Y+Y_{t}\right)\right)+\frac{c_{0}^{2}}{4}\right) V^{2}\right) d Y \geqslant 0 .
$$

This is due to the linear stability of the $1 \mathrm{D}$ wave $\phi_{0}$.

Hence, integral II can be estimated by

$$
\mathrm{II} \leqslant \frac{1}{3}\left(c_{0} \cot \alpha \rho_{1}-\rho_{1}^{2}\right) \int_{\Omega^{\prime}} v^{2}
$$

for $t$ is large enough. 
Because of the choice of $\psi$, the spatial $L^{2}\left(\Omega^{\prime}\right)$ norm of $\psi_{t}$, as well as that of the spatial derivatives of $\psi$ up to second order, is uniformly bounded in $t$. Hence we may, as is classical, estimate III by

$$
\mathrm{III} \leqslant \frac{1}{3}\left(c_{0} \cot \alpha \rho_{1}-\rho_{1}^{2}\right) \int_{\Omega^{\prime}} v^{2}+C_{7}
$$

for some constant $C_{7}$ independent of $t \geqslant 1$.

Summing up (5.11), (5.12) and (5.13), we obtain a uniform control of the $L^{2}$-norm $\|v(t, ., .)\|_{L^{2}\left(\Omega^{\prime}\right)}$, and thus a uniform control of $\left\|v_{1}(t, ., .)\right\|_{L^{2}\left(\Omega^{\prime}\right)}$, for $t$ large enough. Therefore, standard parabolic estimates imply that the function $e^{\rho_{1} X-c_{0} Y / 2} \partial_{e_{\alpha}} \bar{u}=e^{\rho_{1} X-c_{0} Y / 2} \bar{u}_{X}$ is bounded in $L^{\infty}\left(\left(t_{0},+\infty\right) \times \tilde{\Omega}^{\prime}\right)$ for some $t_{0}>0$, where, say, $\tilde{\Omega}^{\prime}=\left\{(x, y) \in \mathbb{R}^{2},(x-1, y) \in\right.$ $\left.\Omega^{\prime}\right\}$. Eventually, since $\left|\nabla_{x, y} \bar{u}\right|$ is globally bounded in $(x, y) \in \mathbb{R}^{2}$ independently of $t \geqslant t_{0}$, one concludes that $e^{\rho_{1} X-c_{0} Y / 2} \bar{u}_{X}$ is bounded in $L^{\infty}\left(\left(t_{0},+\infty\right) \times \mathbb{R}_{+}^{2}\right)$.

Similar estimates can be proven for $\partial_{e_{\alpha}} u(t,-x, y-c t)$. That completes the proof of Proposition 5.2.

Proof of Theorem 1.6. - Step 1. Even if it means shifting both $u_{0}$ and $\phi$, with the same shift, one can assume without loss of generality that $\phi$ is even in $x$ and that

$$
\phi\left(x+x_{n}, y-\left|x_{n}\right| \cot \alpha\right) \rightarrow \phi_{0}( \pm x \cos \alpha+y \sin \alpha)
$$

locally in $(x, y)$ for any sequence $x_{n} \rightarrow \pm \infty$. It then follows from (5.6) that

$$
\left\{\begin{array}{l}
u(t, x+r \sin \alpha, y-r \cos \alpha-c t) \rightarrow \phi_{0}(x \cos \alpha+y \sin \alpha) \\
u(t, x-r \sin \alpha, y-r \cos \alpha-c t) \rightarrow \phi_{0}(-x \cos \alpha+y \sin \alpha)
\end{array} \quad \text { as } r \rightarrow+\infty,\right.
$$

for all $(x, y) \in \mathbb{R}^{2}$ and $t \geqslant 0$ (and also for $t=0$ by assumption on $u_{0}$ ).

Therefore, integrating in $e_{\alpha}$ the bounds given in Proposition 5.2 yields the existence of $C>0$, $t_{0}>0$ such that

$$
\begin{aligned}
& \left|u(t, x, y-c t)-\phi_{0}(x \cos \alpha+y \sin \alpha)\right| \leqslant C e^{-\rho_{1} X+c_{0} Y / 2}, \\
& \left|u(t,-x, y-c t)-\phi_{0}(-x \cos \alpha+y \sin \alpha)\right| \leqslant C e^{-\rho_{1} X+c_{0} Y / 2}
\end{aligned}
$$

for all $t \geqslant t_{0}$ and $(x, y) \in \mathbb{R}_{+}^{2}$.

Since the initial datum $u_{0}:=\phi$ obviously falls within the assumptions of Theorem 1.6, and since $\phi(x, y+c t)$ is the solution of (1.3) with initial condition $\phi$, one concludes that similar estimates as (5.15) also hold with $u(t, \pm x, y-c t)$ replaced with $\phi(x, y)$. Summing (5.15) with these estimates for $\phi$ implies that

$$
\begin{aligned}
& |u(t, x, y-c t)-\phi(x, y)| \leqslant C^{\prime} e^{-\rho_{1}^{\prime} X+c_{0} Y / 2}, \\
& |u(t,-x, y-c t)-\phi(-x, y)| \leqslant C^{\prime} e^{-\rho_{1}^{\prime} X+c_{0} Y / 2}
\end{aligned}
$$

for all $t \geqslant t_{1}$ and $(x, y) \in \mathbb{R}_{+}^{2}$, where $C^{\prime}, t_{1}$ and $\rho_{1}^{\prime}$ are positive constants. 
Therefore, there exists $\rho>0$ (depending only on $\rho_{1}^{\prime}, c_{0}$ and $\alpha$ ), which we may choose less than $\bar{\rho}$ as in Theorem 4.1, such that: for all $\varepsilon>0$ and $y_{1} \in \mathbb{R}$, there is $r \geqslant 0$ such that

$$
\begin{aligned}
& \|u(t, \cdot, \cdot-c t)-\phi\|_{L^{\infty}\left(\mathcal{C}_{-}\left(y_{1}, \alpha\right) \backslash B_{r}\right)} \\
& \quad+\left\|q^{-1}(u(t, \cdot, \cdot-c t)-\phi)\right\|_{L^{\infty}\left(\mathcal{C}_{-}\left(y_{1}, \alpha\right) \backslash B_{r}\right)} \leqslant \varepsilon / 2
\end{aligned}
$$

for all $t \geqslant t_{1}$, where $q$ has been defined in (1.9) and $B_{r}$ denotes the euclidean open ball of center 0 and radius $r$.

Step 2. Let us now prove that $u(t, x, y-c t) \rightarrow \phi(x, y)$ as $t \rightarrow+\infty$ locally uniformly in $(x, y) \in \mathbb{R}^{2}$. If not, there exists a sequence $\left(x_{n}, y_{n}\right) \rightarrow\left(x_{\infty}, y_{\infty}\right) \in \mathbb{R}^{2}$ such that

$$
\liminf _{n \rightarrow+\infty}\left|u\left(t_{n}, x_{n}, y_{n}-c t_{n}\right)-\phi\left(x_{n}, y_{n}\right)\right|>0
$$

From Theorem 1.5, the functions $u\left(t_{n}+t, x, y-c t_{n}-c t\right)$ converge, up to extraction of some subsequence, locally uniformly in $(t, x, y) \in \mathbb{R}^{3}$ to a translate $\tau_{h, k} \phi$ as $n \rightarrow+\infty$. Owing to the definition of $\left(x_{n}, y_{n}\right)$, one has

$$
\left|\tau_{h, k} \phi\left(x_{\infty}, y_{\infty}\right)-\phi\left(x_{\infty}, y_{\infty}\right)\right|>0
$$

On the other hand, the inequalities (5.16) imply, after passage to the limit $t_{n} \rightarrow+\infty$, that

$$
\left|\tau_{h, k} \phi(x, y)-\phi(x, y)\right| \leqslant C^{\prime} e^{-\rho_{1}^{\prime} X+c_{0} Y / 2}
$$

and

$$
\left|\tau_{h, k} \phi(-x, y)-\phi(-x, y)\right| \leqslant C^{\prime} e^{-\rho_{1}^{\prime} X+c_{0} Y / 2}
$$

for all $(x, y) \in \mathbb{R}_{+}^{2}$. It especially follows that $\tau_{h, k} \phi$ and $\phi$ have the same limits along the direction $e_{\alpha}$ and $e_{\alpha}^{\prime}$. Hence $\tau_{h, k} \phi=\phi$, which contradicts (5.18).

Therefore,

$$
u(t, x, y-c t) \rightarrow \phi(x, y) \quad \text { as } t \rightarrow+\infty
$$

locally uniformly in $(x, y) \in \mathbb{R}^{2}$.

Let now $\rho \in(0, \bar{\rho})$ be as in step 1 above. Let $\varepsilon>0$ be any positive number. As already underlined in the proofs of Proposition 5.2 and Theorem 1.5, there exist $y_{2} \geqslant 0$ and $t_{2}>0$ such that

$$
\forall t \geqslant t_{2}, \forall(x, y) \in \mathcal{C}_{+}\left(y_{2}, \pi-\alpha\right), \quad\left\{\begin{array}{l}
\phi(x, y) \geqslant 1-\varepsilon / 8 \\
u(t, x, y-c t) \geqslant 1-\varepsilon / 8 .
\end{array}\right.
$$

Therefore, for all $t \geqslant t_{2}$,

$$
\|u(t, \cdot, \cdot-c t)-\phi\|_{L^{\infty}\left(\mathcal{C}_{+}\left(y_{2}, \pi-\alpha\right)\right)} \leqslant \varepsilon / 4 .
$$

The function $z(t, x, y)=u(t, x, y-c t)-\phi(x, y)$ satisfies the equation

$$
\partial_{t} z-\Delta z+c \partial_{y} z+\gamma(t, x, y) z=0
$$


for some globally bounded function $\gamma$. Without loss of generality, one may also assume that $y_{2}$ and $t_{2}$ are such that

$$
\forall t \geqslant t_{2}, \forall(x, y) \in \mathcal{C}_{+}\left(y_{2}, \pi-\alpha\right), \quad \gamma(t, x, y) \geqslant-\frac{f^{\prime}\left(1^{-}\right)}{2}>0 .
$$

The inequalities (5.17) applied to, say, $\varepsilon / 8$ and $y_{2}$, yield the existence of $r>0$ such that

$$
\forall(x, y) \in \partial \mathcal{C}_{+}\left(y_{2}, \pi-\alpha\right) \backslash B_{r}, \quad|z(t, x, y)| \leqslant \varepsilon q(x, y) / 8
$$

for all $t \geqslant t_{2}^{\prime}$ large enough (we may choose $t_{2}^{\prime} \geqslant t_{2}$ ). Furthermore, one has proved that $z$ converges to 0 locally uniformly as $t \rightarrow+\infty$. Since $q$ is bounded from below in $B_{r}$, one may then assume that $|z(t, x, y)| \leqslant \varepsilon q(x, y) / 8$ for all $t \geqslant t_{2}^{\prime}$ large enough and for all $(x, y) \in B_{r}$. Therefore,

$$
\forall t \geqslant t_{2}^{\prime}, \forall(x, y) \in \partial \mathcal{C}_{+}\left(y_{2}, \pi-\alpha\right), \quad|z(t, x, y)| \leqslant \varepsilon q(x, y) / 8 .
$$

On the other hand, because of (5.6), even if it means decreasing $\rho$ (depending only on $\rho_{0}, \rho_{1}^{\prime}$ and $\alpha$ ), there is a constant $C>0$ such that

$$
\forall(x, y) \in \mathcal{C}_{+}\left(y_{2}, \pi-\alpha\right), \quad\left|z\left(t_{2}^{\prime}, x, y\right)\right|=\left|u\left(t_{2}^{\prime}, x, y-c t_{2}^{\prime}\right)-\phi(x, y)\right| \leqslant C q(x, y) .
$$

Next, the function $h(t, x, y)=\varepsilon q(x, y) / 8+C e^{-\delta\left(t-t_{2}^{\prime}\right)} q(x, y)$ is such that

$$
\partial_{t} h-\Delta h+c \partial_{y} h-\frac{f^{\prime}\left(1^{-}\right)}{2} h \geqslant 0
$$

for $\delta>0$ and $\rho>0$ small enough ( $\rho$ depending only on $c, f^{\prime}\left(1^{-}\right), \alpha,\left\|g^{\prime}\right\|_{\infty}$ and $\left\|g^{\prime \prime}\right\|_{\infty}$ ). Furthermore, $\left|z\left(t_{2}^{\prime}, x, y\right)\right| \leqslant h\left(t_{2}^{\prime}, x, y\right)$ in $\mathcal{C}_{+}\left(y_{2}, \pi-\alpha\right)$, and $|z(t, x, y)| \leqslant h(t, x, y)$ on $\partial \mathcal{C}_{+}\left(y_{2}\right.$, $\pi-\alpha)$ for all $t \geqslant t_{2}^{\prime}$. The maximum principle yields

$$
\forall t \geqslant t_{2}^{\prime}, \forall(x, y) \in \mathcal{C}_{+}\left(y_{2}, \pi-\alpha\right), \quad|z(t, x, y)| \leqslant h(t, x, y)
$$

As a consequence,

$$
\left\|q^{-1}(u(t, \cdot, \cdot-c t)-\phi)\right\|_{L^{\infty}\left(\mathcal{C}_{+}\left(y_{2}, \pi-\alpha\right)\right)} \leqslant \varepsilon / 4
$$

for all $t \geqslant t_{2}^{\prime \prime} \geqslant t_{2}^{\prime}$ large enough.

As a conclusion of this step 2, one has

$$
\begin{aligned}
& \|u(t, \cdot, \cdot-c t)-\phi\|_{L^{\infty}\left(\mathcal{C}_{+}\left(y_{2}, \pi-\alpha\right)\right)} \\
& \quad+\left\|q^{-1}(u(t, \cdot, \cdot-c t)-\phi)\right\|_{L^{\infty}\left(\mathcal{C}_{+}\left(y_{2}, \pi-\alpha\right)\right)} \leqslant \varepsilon / 2
\end{aligned}
$$

for all $t \geqslant t_{2}^{\prime \prime}$.

Step 3 (conclusion). Once $\rho>0$ has been defined in steps 1 and 2, let now $\varepsilon>0$ be as in Theorem 4.1. Let $y_{2}$ be as in step 2, let $y_{1}=y_{2}$ and let $r>0$ be such that (5.17) holds for $t$ large enough. Remember that (5.20) holds for $t$ large enough. Lastly, $u(t, x, y-c t) \rightarrow \phi(x, y)$ locally in $(x, y)$ as $t \rightarrow+\infty$. Since $q$ is bounded from below in $B_{r}$, one gets

$$
\|u(t, \cdot, \cdot-c t)-\phi\|_{L^{\infty}\left(B_{r}\right)}+\left\|q^{-1}(u(t, \cdot, \cdot-c t)-\phi)\right\|_{L^{\infty}\left(B_{r}\right)} \leqslant \varepsilon / 2
$$


for $t$ large enough.

Eventually, there exists $t_{3} \geqslant 0$ such that the function $\tilde{u}_{0}(x, y):=u\left(t_{3}, x, y-c t_{3}\right)$ satisfies: $\tilde{u}_{0}-\phi \in G_{\rho}$ and $\left\|\tilde{u}_{0}-\phi\right\|_{G_{\rho}} \leqslant \varepsilon$. From Theorem 4.1, one concludes that

$$
\forall t \geqslant 0, \quad\left\|u\left(t+t_{3}, \cdot, \cdot-c t-c t_{3}\right)-\phi\right\|_{G_{\rho}} \leqslant K^{\prime} e^{-\omega t}
$$

for some constants $K^{\prime} \geqslant 0$ and $\omega>0$. The conclusion of Theorem 1.6 follows.

Proof of Theorem 1.7. - In $\mathbb{R}_{+}^{2}$ denote, as above, the rotated coordinates by $(X, Y)$. According to the assumptions of the theorem we may define

$$
u_{0,+\infty}(Y)=\lim _{X \rightarrow+\infty} u_{0}(X, Y) .
$$

The function $u_{0,+\infty}$ is such that $\liminf _{Y \rightarrow+\infty} u_{0,+\infty}(Y) \in(\theta, 1]$, and $u_{0,+\infty}(\cdot) \leqslant \phi_{0}\left(\cdot+Y_{0}\right)$ for some $Y_{0} \in \mathbb{R}$, because of the assumptions on $u_{0}$. The solution $u_{+\infty}(t, Y)$ of the Cauchy problem

$$
\begin{aligned}
u_{t}-u_{Y Y}+c_{0} u_{Y} & =f(u) \quad(t>0, Y \in \mathbb{R}) \\
u(0, Y) & =u_{0,+\infty}(Y)
\end{aligned}
$$

converges exponentially in time and uniformly in $Y \in \mathbb{R}$ to a steady 1D solution of the above problem (see [26,34]), which is a 1D wave that we denote by $\phi_{0}\left(Y+Y_{+\infty}\right)$, where $Y_{+\infty} \in \mathbb{R}$.

Fix any sequence $x_{n} \rightarrow+\infty$. The functions $u_{n}(t, x, y)=u\left(t, x+x_{n}, y-\left|x_{n}\right| \cot \alpha-c t\right)$ are bounded in $C_{t}^{1, \delta}\left((0,+\infty) \times \mathbb{R}^{2}\right)$ and $C_{(x, y)}^{2, \delta}\left((0,+\infty) \times \mathbb{R}^{2}\right)$ locally in $(t, x, y) \in(0,+\infty) \times \mathbb{R}^{2}$, for some $\delta>0$. Up to extraction of some subsequence, these functions $u_{n}$ converge locally uniformly in $(0,+\infty) \times \mathbb{R}^{2}$ to a solution $u_{\infty}$ of $\partial_{t} u_{\infty}=\Delta u_{\infty}-c \partial_{y} u_{\infty}+f\left(u_{\infty}\right)$ in $(0,+\infty) \times \mathbb{R}^{2}$.

Fix now any $\varepsilon>0$. Let $v_{0}$ be a function bounded in $C^{3}\left(\mathbb{R}^{2}\right)$ such that $u_{0}-\varepsilon \leqslant v_{0} \leqslant u_{0}+\varepsilon$ in $\mathbb{R}^{2}$ (remember that $u_{0} \in U C\left(\mathbb{R}^{2}\right)$ ), and let $v(t, x, y)$ be the solution of (1.3) with initial condition $v_{0}$. It follows that $\|u(t, \cdot, \cdot)-v(t, \cdot, \cdot)\|_{L^{\infty}\left(\mathbb{R}^{2}\right)} \leqslant \varepsilon e^{\|f\|_{\text {Lip }} t}$ for all $t \geqslant 0$. The functions

$$
v_{n}(t, x, y)=v\left(t, x+x_{n}, y-\left|x_{n}\right| \cot \alpha-c t\right)
$$

converge locally uniformly in $[0,+\infty) \times \mathbb{R}^{2}$ to a solution $v_{\infty}$ of the same equation as $u_{\infty}$, and such that $\left\|u_{\infty}(t, \cdot, \cdot)-v_{\infty}(t, \cdot, \cdot)\right\|_{L^{\infty}\left(\mathbb{R}^{2}\right)} \leqslant \varepsilon e^{\|f\|_{\text {Lip }} t}$ for all $t>0$. Furthermore, one can say from (5.21) that

$$
u_{0,+\infty}(x \cos \alpha+y \sin \alpha)-\varepsilon \leqslant v_{\infty}(0, x, y) \leqslant u_{0,+\infty}(x \cos \alpha+y \sin \alpha)+\varepsilon
$$

for all $(x, y) \in \mathbb{R}^{2}$. Since the function $u_{+\infty}(t, x \cos \alpha+y \sin \alpha)$ is a solution of the equation satisfied by $v_{\infty}$, one then has $\left|v_{\infty}(t, x, y)-u_{+\infty}(t, x \cos \alpha+y \sin \alpha)\right| \leqslant \varepsilon e^{\|f\|_{\text {Lip }} t}$ for all $t \geqslant 0$ and $(x, y) \in \mathbb{R}^{2}$. It follows that $\left|u_{\infty}(t, x, y)-u_{+\infty}(t, x \cos \alpha+y \sin \alpha)\right| \leqslant 2 \varepsilon e^{\|f\|_{\text {Lip }} t}$ for all $t>0$ and $(x, y) \in \mathbb{R}^{2}$. Since $\varepsilon>0$ was arbitrary, one then has that

$$
u_{\infty}(t, x, y) \equiv u_{+\infty}(t, x \cos \alpha+y \sin \alpha) .
$$

By uniqueness of the limit, one concludes that

$$
u(t, x+r \sin \alpha, y-r \cos \alpha) \rightarrow u_{+\infty}(t, Y) \quad \text { as } r \rightarrow+\infty
$$

for all $t \geqslant 0$ and $(x, y) \in \mathbb{R}^{2}$. 
In a similar fashion, we may define $u_{-\infty}\left(t, Y^{\prime}\right)$ and $\phi_{0}\left(Y^{\prime}+Y_{-\infty}\right)$ for the left side, so that $u_{-\infty}\left(t, Y^{\prime}\right) \rightarrow \phi_{0}\left(Y^{\prime}+Y_{-\infty}\right)$ uniformly in $Y^{\prime} \in \mathbb{R}$ as $t \rightarrow+\infty$, and

$$
u(t, x-r \sin \alpha, y-r \cos \alpha) \rightarrow u_{-\infty}\left(t, Y^{\prime}\right) \quad \text { as } r \rightarrow+\infty
$$

for all $t \geqslant 0$ and $(x, y) \in \mathbb{R}^{2}$, where $Y^{\prime}=-x \cos \alpha+y \sin \alpha$.

Under the notations of the proof of Proposition 5.2, the function $\bar{u}_{X}$ satisfies a parabolic linear equation with bounded coefficients. Because of the assumptions on $u_{0}$, it then easily follows that there exists $\omega_{0} \in \mathbb{R}$ such that

$$
\left|\bar{u}_{X}(t, x, y)\right| \leqslant C e^{\rho_{0}(y \sin \alpha-x \cos \alpha)} e^{\omega_{0} t}
$$

for all $t \geqslant 0$ and $(x, y) \in \mathbb{R}^{2}$. Furthermore, step 1 of Proposition 5.2 can be reproduced word by word and it gives some estimates of $\bar{u}_{X}$ in lower cones $\mathcal{C}_{-}\left(y_{0}, \alpha\right)$ for $t \geqslant 1$. Therefore, there exists $\rho_{1}>0$ small enough such that (5.9) holds and the function

$$
v_{1}(t, x, y)=e^{\rho_{1} X-c_{0} Y / 2} \bar{u}_{X}(t, x, y)
$$

is in $L^{2}\left(\Omega^{\prime}\right)$ for each $t \geqslant 1$. To see it, divide $\Omega^{\prime}$ into the following four regions: $\Omega^{\prime} \cap\{X \leqslant 0\}$ (use here the fact that $\bar{u}_{X}$ is globally bounded for $t \geqslant 1$ ), $\Omega^{\prime} \cap\{X \geqslant 0, Y \geqslant \beta X\}$ (use the fact that $\bar{u}_{X}$ is globally bounded in this region for $t \geqslant 1$, and choose $\left.0<\rho_{1}<c_{0} \beta / 2\right), \Omega^{\prime} \cap\{X \geqslant$ $0,|Y| \leqslant \beta X\}$ (use (5.23) and choose $\beta\left(c_{0} / 2+\rho_{0}\left|\sin ^{2} \alpha-\cos ^{2} \alpha\right|\right)+\rho_{1}<2 \rho_{0} \sin \alpha \cos \alpha$ ), and $\Omega^{\prime} \cap\{X \geqslant 0, Y \leqslant-\beta X\}$ (use the estimates in step 1 of Proposition 5.2 and choose again $0<\rho_{1}<c_{0} \beta / 2$ ). Step 6 of Proposition 5.2 can be reproduced and the conclusion of Proposition 5.2 still holds.

Let now $\left(x_{\infty}, y_{\infty}\right)$ be the unique couple of real numbers such that $\tau_{x_{\infty}, y_{\infty}} \phi$ converges to $\phi_{0}\left(Y+Y_{+\infty}\right)$ (respectively $\left.\phi_{0}\left(Y+Y_{-\infty}\right)\right)$ along the direction $e_{\alpha}$ (respectively $e_{\alpha}^{\prime}$ ).

Let us fix any $\varepsilon>0$ and let us prove that

$$
\left\|u(t, \cdot, \cdot-c t)-\tau_{x_{\infty}, y_{\infty}} \phi\right\|_{L^{\infty}\left(\mathbb{R}^{2}\right)} \leqslant \varepsilon
$$

for $t$ large enough.

First of all, as already emphasized, there exists $A \in \mathbb{R}$ such that $1-\varepsilon / 2 \leqslant u(t, x, y-c t) \leqslant 1$ in $\mathcal{C}_{+}(A, \pi-\alpha)$ for $t$ large enough, and $1-\varepsilon / 2 \leqslant \tau_{x_{\infty}, y_{\infty}} \phi \leqslant 1$ in $\mathcal{C}_{+}(A, \pi-\alpha)$, whence

$$
\left\|u(t, \cdot, \cdot-c t)-\tau_{x_{\infty}, y_{\infty}} \phi\right\|_{L^{\infty}\left(\mathcal{C}_{+}(A, \pi-\alpha)\right)} \leqslant \varepsilon
$$

for $t$ large enough. Similarly, since $0 \leqslant u(t, x, y-c t) \leqslant \phi(x, y)$ (because $0 \leqslant u_{0} \leqslant \phi$ ), there exists $B \leqslant A$ such that

$$
\left\|u(t, \cdot, \cdot-c t)-\tau_{x_{\infty}, y_{\infty}} \phi\right\|_{L^{\infty}\left(\mathcal{C}_{-}(B, \alpha)\right)} \leqslant \varepsilon
$$

for all $t \geqslant 0$.

Let $S=\mathcal{C}_{+}(A, \pi-\alpha) \backslash \mathcal{C}_{-}(B, \alpha)$. Because of the estimates for $\bar{u}_{X}$ as in the conclusion of Proposition 5.2, and because of (5.22), there exists $t_{+} \geqslant 0$ and $x_{+} \geqslant 0$ such that

$$
\left|u(t, x, y-c t)-u_{+\infty}(t, Y)\right| \leqslant \varepsilon / 3
$$

for all $t \geqslant t_{+}$and for all $(x, y) \in S \cap\left\{x \geqslant x_{+}\right\}$. On the other hand,

$$
\left\|u_{+\infty}(t, \cdot)-\phi_{0}\left(\cdot+Y_{+\infty}\right)\right\|_{L^{\infty}(B / \sin \alpha, A / \sin \alpha)} \leqslant \varepsilon / 3
$$


for $t$ large enough. Lastly, even if it means increasing $x_{+}$, one can assume that

$$
\left|\tau_{x_{\infty}, y_{\infty}} \phi(x, y)-\phi_{0}\left(Y+Y_{+\infty}\right)\right| \leqslant \varepsilon / 3
$$

for all $(x, y) \in S \cap\left\{x \geqslant x_{+}\right\}$. Therefore,

$$
\left\|u(t, \cdot, \cdot-c t)-\tau_{x_{\infty}, y_{\infty}} \phi\right\|_{L^{\infty}\left(S \cap\left\{x \geqslant x_{+}\right\}\right)} \leqslant \varepsilon
$$

for $t$ large enough.

Similarly, there exists $x_{-} \leqslant 0$ such that

$$
\left\|u(t, \cdot, \cdot-c t)-\tau_{x_{\infty}, y_{\infty}} \phi\right\|_{L^{\infty}\left(S \cap\left\{x \leqslant x_{-}\right\}\right)} \leqslant \varepsilon
$$

for $t$ large enough.

Lastly, from Theorem 1.5 , there exist a sequence $t_{n} \rightarrow+\infty$ and $(h, k) \in \mathbb{R}^{2}$ such that

$$
u\left(t_{n}, x, y-c t_{n}\right) \rightarrow \tau_{h, k} \phi(x, y)
$$

locally uniformly in $(x, y) \in \mathbb{R}^{2}$ as $n \rightarrow+\infty$. The arguments above prove that for each $\varepsilon^{\prime}>0$, there exists $R=R_{\varepsilon^{\prime}} \geqslant 0$ such that $\left\|\tau_{h, k} \phi-\tau_{x_{\infty}, y_{\infty}} \phi\right\|_{L^{\infty}\left(\mathbb{R}^{2} \backslash B_{R}\right)} \leqslant \varepsilon^{\prime}$. As a consequence, $\tau_{h, k} \phi$ and $\tau_{x_{\infty}, y_{\infty}} \phi$ have the same limits along the directions $e_{\alpha}$ and $e_{\alpha}^{\prime}$, whence $\tau_{h, k} \phi=\tau_{x_{\infty}, y_{\infty}} \phi$. Hence, by uniqueness of the limit, one can say that the whole family $u(t, x, y-c t)$ converges to $\tau_{x_{\infty}, y_{\infty}} \phi$ as $t \rightarrow+\infty$, locally uniformly in $(x, y) \in \mathbb{R}^{2}$.

Eventually, one concludes that

$$
\left\|u(t, \cdot, \cdot-c t)-\tau_{x_{\infty}, y_{\infty}} \phi\right\|_{L^{\infty}\left(S \cap\left\{x_{-} \leqslant x \leqslant x_{+}\right\}\right)} \leqslant \varepsilon
$$

for $t$ large enough.

As a conclusion,

$$
\left\|u(t, \cdot, \cdot-c t)-\tau_{x_{\infty}, y_{\infty}} \phi\right\|_{L^{\infty}\left(\mathbb{R}^{2}\right)} \leqslant \varepsilon
$$

for $t$ large enough. That completes the proof of Theorem 1.7.

\section{Acknowledgements}

The authors are grateful to H. Berestycki, A. Bonnet, L.A. Caffarelli, for helpful comments and discussions during the preparation of this paper. Part of this work was done by the second author at the Massachusetts Institute of Technology with the support of a NATO grant; this author is indebted to these two institutions. The third author was partially supported by the CNRS Young Researcher Grant 'Combustion diphasique'.

\section{REFERENCES}

[1] Agmon S., Nirenberg L., Properties of solutions of ordinary differential equations in Banach space, Comm. Pure Appl. Math. 16 (1963) 121-239.

[2] Aronson D.G., Weinberger H.F., Nonlinear diffusion in population genetics, combustion and nerve propagation, in: Part. Diff. Eq. and Related Topics, in: Lecture Notes in Math., vol. 446, Springer, New York, 1975, pp. 5-49. 
[3] Berestycki H., Hamel F., Front propagation in periodic excitable media, Comm. Pure Appl. Math. 55 (2002) 949-1032.

[4] Berestycki H., Larrouturou B., Lions P.-L., Multidimensional travelling-wave solutions of a flame propagation model, Arch. Rat. Mech. Anal. 111 (1990) 33-49.

[5] Berestycki H., Larrouturou B., Roquejoffre J.-M., Stability of travelling fronts in a curved flame model, Part I, Linear analysis, Arch. Rat. Mech. Anal. 117 (1992) 97-117.

[6] Berestycki H., Nicolaenko B., Scheurer B., Traveling waves solutions to combustion models and their singular limits, SIAM J. Math. Anal. 16 (6) (1985) 1207-1242.

[7] Berestycki H., Nirenberg L., On the method of moving planes and the sliding method, Bol. Soc. Bras. Mat. 22 (1991) 1-37.

[8] Berestycki H., Nirenberg L., Travelling fronts in cylinders, Ann. Inst. H. Poincaré, Anal. Non Lin. 9 (1992) 497-572.

[9] Bonnet A., HAMEL F., Existence of non-planar solutions of a simple model of premixed Bunsen flames, SIAM J. Math. Anal. 31 (1999) 80-118.

[10] Bramson M., Convergence of Solutions of the Kolmogorov Equation to Travelling Waves, in: Mem. Amer. Math. Soc., vol. 44, 1983.

[11] BRAZHNiK P.K., TYSON J.J., On traveling wave solutions of Fisher's equation in two spatial dimensions, SIAM J. Appl. Math. 60 (2000) 371-391.

[12] BuCKMASter J.D., LudFord G.S.S., Lectures on Mathematical Combustion, in: CBMS-NSF Conf. Series in Applied Math., vol. 43, SIAM, 1983.

[13] Caffarelli L.A., CABré X., Fully Nonlinear Elliptic Equations, in: Colloquium Publications, in: Amer. Math. Soc., vol. 43, 1995.

[14] Crandall M.G., Ishit H., LiOns P.-L., User's guide to viscosity solutions of second order partial differential equations, Bull. Amer. Math. Soc. (N.S.) 27 (1992) 1-67.

[15] Collet P., EcKmann J.-P., Space-time behaviour in problems of hydrodynamic type: a case study, Nonlinearity 5 (6) (1992) 1265-1302.

[16] FIFE P.C., Dynamics of internal layers and diffusive interfaces, in: Cbms-Nsf Regional Conference, in: Series in Applied Mathematics, vol. 53, 1988.

[17] FIFE P.C., MCLEOD J.B., The approach of solutions of non-linear diffusion equations to travelling front solutions, Arch. Rat. Mech. Anal. 65 (1977) 335-361.

[18] Gilbarg D., Trudinger N.S., Elliptic Partial Differential Equations of Second Order, SpringerVerlag, 1997.

[19] Hamel F., Monneau R., Solutions of semilinear elliptic equations in $\mathbb{R}^{N}$ with conical-shaped level sets, Comm. Partial Differential Equations 25 (2000) 769-819.

[20] Hamel F., Monneau R., Existence and uniqueness for a free boundary problem arising in combustion theory, Interfaces Free Boundaries 4 (2002) 167-210.

[21] Hamel F., NAdiRAshvili N., Travelling waves and entire solutions of the Fisher-KPP equation in $\mathbb{R}^{N}$, Arch. Ration. Mech. Anal. 157 (2001) 91-163.

[22] Henry D., Geometric Theory of Semilinear Parabolic Equations, in: Lecture Notes in Math., Springer Verlag, New York, 1981.

[23] IsHII H., On uniqueness and existence of viscosity solutions of fully nonlinear second-order elliptic PDE's, Comm. Pure Appl. Math. 42 (1989) 15-45.

[24] Joulin G., Dynamique des fronts de flammes, in: Modélisation de la combustion, Images des Mathématiques, CNRS, 1996.

[25] Kanel' YA.I., Certain problems of burning-theory equations, Sov. Math. Dokl. 2 (1961) 48-51.

[26] KANEL' YA.I., Stabilization of solution of the Cauchy problem for equations encountered in combustion theory, Mat. Sbornik 59 (1962) 245-288.

[27] Levermore C.D., XIN J.X., Multidimensional stability of travelling waves in a bistable reactiondiffusion equation, II, Comm. Partial Differential Equations 17 (1992) 1901-1924.

[28] Lewis B., Von Elbe G., Combustion, Flames and Explosions of Gases, Academic Press, New York, 1961.

[29] Mallordy J.-F., RoQuejoffre J.-M., A parabolic equation of the KPP type in higher dimensions, SIAM J. Math. Anal. 26 (1995) 1-20. 
[30] Matkowsky B.J., Olagunju D.O., Pulsations in a burner-stabilized premixed plane flame, SIAM J. Appl. Math. 40 (1981) 551-562.

[31] MiCHELSON D., Stability of the Bunsen flame profiles in the Kuramoto-Sivashinsky equation, SIAM J. Math. Anal. 27 (1996) 765-781.

[32] Ninomiya H., TANiguchi M., Stability of traveling curved fronts in a curvature flow with driving force, Meth. Appl. Anal., submitted for publication.

[33] RoQUEJOFFre J.-M., Stability of travelling fronts in a curved flame model, Part II: Non-linear orbital stability, Arch. Rat. Mech. Anal. 117 (1992) 119-153.

[34] RoQUEJOFFrE J.-M., Convergence to travelling waves for solutions of a class of semilinear parabolic equation, J. Differential Equations 108 (1994) 262-295.

[35] RoQUEJOFFRE J.-M., Eventual monotonicity and convergence to travelling fronts for the solutions of parabolic equations in cylinders, Ann. Inst. H. Poincaré, Anal. Non Lin. 14 (1997) 499-552.

[36] SATtinger D.H., Stability of waves of nonlinear parabolic systems, Adv. Math. 22 (1976) 312-355.

[37] SATTINGER D.H., Weighted norms for the stability of travelling waves, J. Differential Equations 25 (1977) 130-144.

[38] Sivashinsky G.I., The structure of Bunsen flames, J. Chem. Phys. 62 (1975) 638-643.

[39] STEWART H.B., Generation of analytic semigroups by strongly elliptic operators under general boundary conditions, Trans. Amer. Math. Soc. 259 (1980) 299-310.

[40] Williams F., Combustion Theory, Addison-Wesley, Reading, MA, 1983.

[41] XIN J.X., Muldimensional stability of travelling waves in a bistable reaction-diffusion equation, I, Comm. Partial Differential Equations 17 (1992) 1889-1899.

(Manuscrit reçu le 18 juin 2003 ; accepté le 5 mars 2004.)

\author{
François HAMEL \\ LATP (UMR CNRS 6632), \\ Université Aix-Marseille III, \\ Faculté Saint-Jérôme, \\ Avenue Escadrille Normandie-Niemen, \\ 13397 Marseille Cedex 20, France \\ E-mail: francois.hamel@univ.u-3mrs.fr
}

\author{
Régis MonNEAU \\ CERMICS-ENPC, \\ 6-8 avenue B. Pascal, Cité Descartes, \\ 77455 Marne-La-Vallée Cedex 2, France \\ E-mail: monneau@cermics.enpc.fr \\ Jean-Michel ROQUEJOFFRE \\ Laboratoire M.I.P. (UMR CNRS 5640), \\ et Institut Universitaire de France, \\ Université Paul Sabatier, 118 route de Narbonne, \\ 31062 Toulouse Cedex 4, France \\ E-mail: roque@mip.ups-tlse.fr
}

